Control of electron energy distributions and plasma characteristics of dual frequency, pulsed capacitively coupled plasmas sustained in $\mathrm{Ar}$ and $\mathrm{Ar} / \mathrm{CF}_{4} / \mathrm{O}_{2}$

This article has been downloaded from IOPscience. Please scroll down to see the full text article.

2012 Plasma Sources Sci. Technol. 21055028

(http://iopscience.iop.org/0963-0252/21/5/055028)

View the table of contents for this issue, or go to the journal homepage for more

Download details:

IP Address: 141.211.173.82

The article was downloaded on 25/06/2013 at 20:32

Please note that terms and conditions apply. 


\title{
Control of electron energy distributions and plasma characteristics of dual frequency, pulsed capacitively coupled plasmas sustained in $\mathrm{Ar}$ and $\mathrm{Ar} / \mathrm{CF}_{4} / \mathrm{O}_{2}$
}

\author{
Sang-Heon Song ${ }^{1}$ and Mark J Kushner ${ }^{2}$ \\ ${ }^{1}$ Department of Nuclear Engineering and Radiological Sciences, University of Michigan, 2355 Bonisteel \\ Boulevard, Ann Arbor, MI 48109, USA \\ ${ }^{2}$ Department of Electrical Engineering and Computer Science, University of Michigan, 1301 Beal \\ Avenue, Ann Arbor, MI 48109, USA \\ E-mail: mjkush@umich.edu
}

Received 14 April 2012, in final form 4 September 2012

Published 27 September 2012

Online at stacks.iop.org/PSST/21/055028

\begin{abstract}
The fluxes of radicals and ions to the wafer during plasma processing of microelectronics devices determine the quality of the etch or deposition. These fluxes are largely controlled by controlling the electron energy distribution function $f(\varepsilon)$ which determines the dissociation patterns of feedstock gases. In quasi-steady state operation, an equilibrium condition for $f(\varepsilon)$ results from a real time balance between electron sources and sinks. Using pulsed power, electron sources and sinks do not need to instantaneously balance-they only need to balance over the longer pulse period. This provides additional leverage to customize $f(\varepsilon)$. In this paper, the $f(\varepsilon)$ in a two-frequency, pulsed capacitively coupled plasma sustained in Ar and $\mathrm{Ar} / \mathrm{CF}_{4} / \mathrm{O}_{2}$ mixtures are discussed with results from a two-dimensional plasma hydrodynamics model. The $f(\varepsilon)$ are obtained from a Monte Carlo simulation which includes electron-electron collisions. We found that the $f(\varepsilon)$ and rate coefficients can be controlled by pulse repetition frequency (PRF) and duty cycle (DC) of the pulsed power in a manner not otherwise easily attainable using continuous excitation. The tail of the $f(\varepsilon)$ is enhanced with smaller PRF and $\mathrm{DC}$ in order to compensate for the electron losses during the power-off portion of the cycle.
\end{abstract}

(Some figures may appear in colour only in the online journal)

\section{Introduction}

In capacitively coupled radio frequency (rf) discharges, as used in plasma processing of semiconductors, controlling the electron energy distribution, $f(\varepsilon)$, is important to controlling the flux of radicals and ions to the substrate [1]. $f(\varepsilon)$ of electrons in plasma sources as typically used in materials processing have been extensively investigated $[2,3]$ and, given electric fields and gas mixtures, are generally predictable. However, there are emerging needs for better controlling $f(\varepsilon)$ in order to, for example, optimize the production of a particular radical. There have been several attempts at controlling $f(\varepsilon)$, including tuning the gas pressure [4], adding an external ionization sources such as an electron beam [5], using magnetic fields [6], using an augmenting direct current bias on an rf electrode [7] and varying the frequency [8].

An additional parameter that may be used to customize $f(\varepsilon)$ is using pulsed power [9]. In quasi-steady state operation, an equilibrium condition for $f(\varepsilon)$ requires an instantaneous (or rf cycle average) balance between electron sources and sinks. As such, for a given geometry, pressure and frequency of operation, there is little latitude in customizing $f(\varepsilon)$. Using pulsed power, electron sources and sinks do not need to instantaneously balance-they only need to balance averaged over the longer pulsed period. This provides additional leverage to control $f(\varepsilon)$. By pulsing, one may be able to modulate $f(\varepsilon)$ to produce shapes or access energies that are not otherwise (or easily) attainable using continuous wave 
$(\mathrm{CW})$ excitation. For example, $f(\varepsilon)$ may be produced that has both a high-energy tail and a large thermal component. These $f(\varepsilon)$ will produce different dissociation patterns of the feedstock gases and so produce different ratios of fluxes to the substrate for a given time average power. This strategy of customizing fluxes using pulsed power has been applied in inductively coupled plasmas (ICPs) [10].

For example, during the power-off period of a pulsed cycle, high-energy electrons may quickly thermalize due to inelastic collisions and be lost by rapid diffusion to the wall. At the beginning of the power-on portion of the pulsed cycle, high-energy electrons are generated due to an overshoot of $E / N$ (electric field/gas number density) above the value that can be sustained in the steady state. This overshoot is due to the initially lower conductivity of the plasma following electron losses during the power-off period. In such systems, $f(\varepsilon)$ can be controlled through choice of the pulse repetition frequency (PRF) and duty cycle (DC). (DC is the fraction of the pulsed period that power is applied.) These determine the relative roles of both electron acceleration during the power-on portion of the cycle and thermalization during the power-off portion. These concepts have been demonstrated in pulsed ICPs to produce ion-ion plasmas during the power-off period, and so provide a means for negative ion acceleration into trenches to remediate charge damage [11]. Pulsed capacitively coupled plasmas (CCPs) are a more recent development [12].

In this paper, we build on these prior works using results from a computational investigations to discuss strategies for controlling $f(\varepsilon)$ of electrons by varying the PRF and DC in CCPs. We found that the tail of $f(\varepsilon)$ is more enhanced when operating with a lower PRF in order to compensate for the losses of electrons incurred during the longer afterglow period. For this reason, the overshoot of the tail of $f(\varepsilon)$ at the beginning of the power-on period is particularly prominent in $\mathrm{Ar} / \mathrm{CF}_{4} / \mathrm{O}_{2}$ gas mixtures compared with Ar mixtures due to the larger electron losses during the power-off period. Due to the transient nature of pulsed CCPs in which the electron temperature cycles from above the steady-state value to below, the relative rate of attachment is particularly high in these mixtures during the power-off period. Secondary electrons emitted from surfaces and accelerated in the sheath provide the additional ionization required to sustain the plasma in electronegative gas mixtures where electron attachment and dissociative recombination dominate at low energies. Varying the rate of secondary-electron emission then provides an additional means for controlling $f(\varepsilon)$.

The model used in this study is described in section 2 with emphasis on the computation of $f(\varepsilon)$. The $f(\varepsilon)$ in pulsed dual frequency capacitively coupled plasma (DF-CCP) sustained in Ar are discussed in section 3, and sustained in $\mathrm{Ar} / \mathrm{CF}_{4} / \mathrm{O}_{2}$ are discussed in section 4. A comparison of ionization and loss rates between $\mathrm{Ar}$ and $\mathrm{Ar} / \mathrm{CF}_{4} / \mathrm{O}_{2}$ is presented in section 5. Our concluding remarks are in section 6 .

\section{Description of the model and gas phase reaction mechanism}

The model used in this investigation is a two-dimensional fluid hydrodynamics simulation in which the electron energy distributions of bulk and secondary electrons are obtained using an electron Monte Carlo simulation (eMCS). The model is described in detail in [13]. Briefly, continuity, momentum and energy equations for neutrals and ions, continuity equations for electrons and Poisson's equation for the electric potential are integrated in time to obtain a periodic steady state. The computational module in which these calculations are performed is the fluid kinetics simulation (FKS). The resulting electric fields and ion fluxes to surfaces are periodically transferred to the eMCS where the electron energy transport of bulk and secondary electrons emitted from surfaces is addressed. Electron-impact source functions and sources of secondary-electron current are derived from these distribution functions and are returned to the fluid model. The process is iterated to convergence.

The electron energy distributions as a function of position, $f(\varepsilon, r)$, are obtained using the eMCS, which is a statistical, kinetic solution of Boltzmann's equation. The eMCS, including our algorithms for electron-electron collisions, is described in detail in [14]. The description here is an update and summary of that discussed in [14].

The eMCS is a $3 \mathrm{v}-3 \mathrm{~d}$ (3 velocity components, 3 dimensions) model which integrates electron trajectories in electric fields obtained from the fluid modules of the model, and employs Monte Carlo techniques for collisions with heavy particles and with other electrons. In the fluid portion of the model, charge densities and Poisson's equation are integrated as a function of time over many rf cycles. Over the last cycle of integration prior to calling the eMCS, the vector components of the electric field are recorded as a function of position and phase during the rf cycle, $\vec{E}(\vec{r}, \phi)$. These recordings typically contain 200 phase points during the lower frequency rf cycle and are recorded on the same spatial mesh as the fluid portion of the model is performed. The cycle-averaged densities of all charged and neutral species, $N_{i}(\vec{r})$, are also recorded. These electric fields and densities are transferred to the eMCS. In the eMCS, two simulations are performed-for bulk and for secondary beam electrons.

For the computation of the distribution function of bulk electrons, $f_{\mathrm{b}}(\varepsilon, \vec{r})$, at the beginning of the first call to the eMCS, electrons are initially given a Maxwellian velocity distribution and placed in the reactor using a distribution weighted by the local electron density obtained from the fluid simulation. On subsequent calls to the eMCS, the trajectories are restarted from their coordinates at the end of the previous call to the eMCS. The trajectories of pseudoparticles are advanced using a second-order Euler method. For integration of the trajectory (location $\vec{r}$ and velocity $\vec{v}$ ) of a pseudoparticle from $t$ to $t+\Delta t$,

$$
\begin{gathered}
\vec{r}^{\prime}(t+\Delta t)=\vec{r}(t)+\vec{v}(t) \Delta t, \\
\vec{v}^{\prime}(t+\Delta t)=\vec{v}(t)+\frac{q \vec{E}(\vec{r}(t), \phi(t))}{m_{\mathrm{e}}} \Delta t, \\
\vec{r}(t+\Delta t)=\vec{r}(t)+\frac{\Delta t}{2}\left(\vec{v}(t)+\vec{v}^{\prime}(t+\Delta t)\right), \\
\vec{v}(t+\Delta t)=\vec{v}(t)+\frac{q \Delta t}{2 m_{\mathrm{e}}}(\vec{E}(\vec{r}(t), \phi(t)) \\
\left.+\vec{E}\left(\vec{r}^{\prime}(t+\Delta t), \phi(t+\Delta t)\right)\right),
\end{gathered}
$$


where $\vec{E}(\vec{r}(t), \phi(t))$ at an arbitrary position and time is obtained from a second-order interpolation of the record of $\vec{E}(\vec{r}, \phi)$ on the numerical mesh at fixed phase points. In this investigation, the calculation of densities and solution of Poisson's equation in the fluid portion of the model are performed in cylindrical coordinates with azimuthal symmetry, $(r, z)$. For computational convenience to more easily facilitate the collision operator, the integration of trajectories in the eMCS is performed in 3D Cartesian coordinates. The cylindrical vector components of $\vec{E}(r, z, \phi)$ are transformed to Cartesian vector components $\vec{E}(x, y, z, \phi)$ based on the instantaneous position of each particle.

Since Poisson's equation is not being solved in the eMCS, the particles do not need to be at the same time unless statistics are being collected. Therefore, the time step $\Delta t$ for each particle is independently chosen as the minimum of the following: a specified fraction of the rf cycle, the time to cross half of the computational mesh in any direction, the time to the next collision, the time for the particle to be decelerated to zero speed, or the time to when statistics are being collected when all particles should be at the same time, $T_{\mathrm{f}}$. Once a particle has reached $T_{\mathrm{f}}$, its trajectory is no longer integrated until other particles reach $T_{\mathrm{f}}$. This is carried out in a computational efficient manner by stenciling out particles having already reached $T_{\mathrm{f}}$ and shuffling the arrays containing particle information so that there is a (nearly) continuous array of particles whose trajectories are being integrated. This enables more efficient pipelining of the numerical operations. After recording statistics, the trajectories are restarted. (In the case of two-frequency excitation with the lower frequency being $10 \mathrm{MHz}$ and the higher frequency being $40 \mathrm{MHz}$, the time step is limited to be no greater than $0.5 \%$ of the lowfrequency (LF) cycle and $2 \%$ of the high-frequency (HF) cycle.)

Statistics and collision frequencies are discretely collected or calculated on an energy grid. Energy bins have constant widths over a specified energy range to simplify gathering statistical data while resolving the structure in electron-impact cross sections. In this work, 500 total bins were used with energy ranges (100 bins/range) 0-5, 5-12, 12-50, 50-300 and $300-1000 \mathrm{eV}$. (The extended range in energy is used to cover the energy of sheath accelerated secondary electrons.) Within energy bin $i$, the total collision frequency, $v_{i}$, is computed by summing all the possible collisions with heavy particles,

$$
v_{i}=\left(\frac{2 \varepsilon_{i}}{m_{\mathrm{e}}}\right)^{1 / 2} \sum_{j, k} \sigma_{i j k} N_{j},
$$

where $\varepsilon_{i}$ is the average energy within the bin, $\sigma_{i j k}$ is the cross section at energy $i$, for species $j$ and collision process $k$, and $N_{j}$ is the number density of species $j$. As this point, $v_{i}$ does not account for the frequency of electron-electron (e-e) collisions since this frequency depends on the relative velocity of the collision partners and, therefore, depends on the dynamics of these trajectories during the simulation.

Separate null collision cross sections are used in each energy range to provide a constant collision frequency. This is accomplished by adding an additional fictitious process referred to as a null collision such that all electrons within a given energy range appear to have the same collision frequency [15]. The null collisional frequency at energy $\varepsilon_{i}$ in energy range $j$ is $v_{n i j}=v_{m j}-v_{i}$, where $v_{m j}$ is the maximum collision frequency in energy range $j$ based on both electron energy and density of collision partners. The separate null collision frequency in each energy range is used to minimize the occurrence of null collisions since over the range of expected electron energies, the total collision frequency can vary by more than an order of magnitude. The time between collisions is obtained from $\Delta t=-\ln \left(r_{1}\right) / v_{m j}$, where $r_{1}$ is a random number distributed on $(0,1)$. There is an inconsistency in choosing $\Delta t$ if, between collisions, the particle crosses the boundary between energy ranges and $v_{m j}$ varies between ranges. However, the frequency of these occurrences is small. The type of collision is determined by generating a series of random numbers. If $r_{2} \leqslant v_{n i j} / v_{m j}$, then the collision is null and the electron trajectory continues unhindered. For a real collision, we find the particular electron collision $j$ which satisfies

$$
\frac{1}{v_{m j}} \sum_{k=1}^{n-1} v_{k}<r_{3} \leqslant \frac{1}{v_{m j}} \sum_{k=1}^{n} v_{k}
$$

where all collision frequencies are computed based on the maximum density of the collision partner in the entire reactor for process $n, N_{m n}$. A second level of null collision is then used to determine if based on the local density of the collision partner a real or null collision has occurred. If $r_{4} \leqslant N_{n}(\vec{r}) / N_{m n}$, where $N_{n}(\vec{r})$ is the actual local density of the collision partner, then a real collision occurs. Otherwise, the collision is considered null and the trajectory proceeds unhindered.

After determining the final type of collision, the electron energy is reduced according to the inelastic or elastic nature of the collision (or increased in the case of a superelastic collision), and the trajectory is scattered. The final velocity following a collision is determined by applying the scattering matrix,

$$
\begin{aligned}
& \begin{aligned}
v_{x}= & v \cdot(\cos \beta \cdot \cos \alpha \cdot \sin \theta \cdot \cos \phi+\cos \beta \cdot \sin \alpha \cdot \cos \theta \\
& \quad-\sin \beta \cdot \sin \theta \cdot \sin \phi) \\
v_{y}= & v \cdot(\sin \beta \cdot \cos \alpha \cdot \sin \theta \cdot \cos \phi+\sin \beta \cdot \sin \alpha \cdot \cos \theta \\
& \quad+\cos \beta \cdot \sin \theta \cdot \sin \phi) \\
v_{z}= & v \cdot(-\sin \alpha \cdot \sin \theta \cdot \cos \phi+\cos \alpha \cdot \cos \theta),
\end{aligned}
\end{aligned}
$$

where $\alpha$ and $\beta$ are the polar and azimuthal Eulerian angles of the electron velocity prior to the collision; $\theta$ and $\phi$ are the polar and azimuthal scattering angles, and $v$ is the electron speed after the collision. Assuming azimuthal symmetry for the collision, $\phi$ is randomly chosen from the interval $(0,2 \pi)$. Unless experimental data are available, $\theta$ is chosen by specifying a scattering parameter $\chi$ where the polar scattering probability is proportional to $\cos ^{\chi}(\theta / 2) . \quad \chi=0$ provides for isotropic scattering and $\chi \gg 1$ provides for forward scattering. The randomly selected scattering angle is then

$$
\theta=2\left[\cos ^{-1}\left(1-r_{5}\right)\right]^{(1 /(2+\chi))} .
$$

In the absence of experimental data, we used $\chi=0-0.1$ for elastic collisions derived from momentum transfer collision cross sections. For inelastic collisions, $\chi=2-3$. 
Following an ionizing collision, a secondary electron is added to the simulation at the same location as the primary particle and with a randomly chosen isotropic angular distribution. The distribution of secondary energies, $f_{\mathrm{sec}}(\varepsilon)$, produced by an ionizing collision with species $j$ by primary electron with energy $\varepsilon_{\mathrm{p}}$ is randomly chosen from [16]:

$$
\begin{aligned}
f_{\mathrm{sec}}(\varepsilon) & \sim\left(1+\left(\frac{\varepsilon}{\bar{\varepsilon}_{j}}\right)^{2}\right)^{-1}, \\
\varepsilon & =\bar{\varepsilon}_{j} \tan \left(r_{6} \cdot \tan \left(\frac{\varepsilon_{\mathrm{p}}-E_{j}}{\bar{\varepsilon}_{j}}\right)^{-1}\right),
\end{aligned}
$$

where $\bar{\varepsilon}_{j}$ is a semi-empirical parameter for species $j$ having ionization potential $E_{j}$.

Statistics for $f_{\mathrm{b}}(\varepsilon)$ are collected for every particle on every time step. The particles are binned by energy and location with a weighting proportional to the product of the number of electrons each pseudoparticle represents, $w$, and the time spent in the spatial mesh cell, $\Delta t$. Finite particle size techniques are used to distribute the particle weighting to its own cell and to neighboring cells in proportion to the fraction of the volume of the finite particle size that resides in the neighboring cell, $\eta$. The particle size and volume are equal to that of the numerical mesh. So for a particle in spatial bin $j$ and energy bin $i$, the running sum of statistics is

$$
\sum_{j^{\prime}=j, \text { neighbors }}\left[F_{i j^{\prime}} \rightarrow F_{i j^{\prime}}+w \Delta t \eta_{j j^{\prime}}\right] .
$$

When modeling transients, the time spent in the FKS between calls to the eMCS is relatively short so that there is frequent feedback from the eMCS to the fluid modules and vice versa. The relative change in voltage or power should be small between calls to the eMCS. To maintain the eMCS in lockstep with the fluid simulations, in this study trajectories are computed for $5 \mathrm{rf}$ LF cycles for each call of the eMCS (which at $10 \mathrm{MHz}$ is $0.5 \mu \mathrm{s}$ ). Statistics are typically gathered for only the latter two or three of those cycles to allow for artificial transients which may occur at the beginning of each iteration to dampen out. An average of 50000 pseudoparticles are used, with particles added for ionizations and removed for losses by attachment, recombination or leaving the volume. If the particle number exceeds a maximum value (typically 150000 ), then the particle number is reduced by randomly removing particles. If the particle number is reduced below a minimum value (typically 40000 ), particles are randomly seeded in the plasma. When a particle is removed from the simulation, its indice in arrays for location and velocity is stenciled out - the location is termed empty. A record is kept of the empty locations and new particles are first placed into the empty locations while keeping track of the highest indice in the array that is occupied. If the fraction of empty locations exceeds a specified value (which may be computer dependent), the empties are removed by compressing the stack of arrays so that pipelining can be more efficiently performed.

At the end of a given call to the eMCS, the $f_{\mathrm{b}}(\varepsilon, \vec{r})$ at each spatial location is obtained by normalizing the statistics such that

$$
\sum_{i} F_{i j}=\sum_{i} f_{\mathrm{b} i j} \varepsilon_{i}^{1 / 2} \Delta \varepsilon_{i}=1,
$$

where $f_{\mathrm{b} i j}\left(\mathrm{eV}^{-3 / 2}\right)$ is the $f_{\mathrm{b}}\left(\varepsilon_{i}, \vec{r}\right)$ at $\vec{r}$, and $\Delta \varepsilon_{i}$ is the width of the energy bin.

e-e collisions are accounted for using a particle mesh technique where the electrons collide with an energy-resolved electron fluid. This is accomplished using spatially dependent $f_{\mathrm{b}}(\varepsilon, \vec{r})$ recorded during the previous call to the eMCS. The incident pseudoparticle in the e-e collision begins with a velocity $\vec{v}_{0}$. The velocity of an electron collision partner for the incident pseudoparticle is randomly chosen from the distribution function at that location, $f_{\mathrm{b}}(\varepsilon, \vec{r})$, that was computed on the previous call to the eMCS. As only the energy distribution $f_{\mathrm{b}}(\varepsilon, \vec{r})$ is retained from the previous iteration, as opposed to the electron velocity distribution, we assume that the chosen target electron has an isotropic angular distribution. The probability of selecting a collision partner having an energy $\varepsilon^{\prime}$ for a pseudoparticle in the $j$ th spatial bin is determined with a cumulative probability. With,

$$
P_{j}\left(\varepsilon^{\prime}\right)=f_{j}\left(\varepsilon^{\prime}\right) \varepsilon^{1 / 2} \Delta \varepsilon^{\prime} /\left(\sum_{i} f_{j}\left(\varepsilon_{i}^{\prime}\right) \varepsilon_{i}^{1 / 2} \Delta \varepsilon^{\prime}\right),
$$

where $f_{j}\left(\varepsilon^{\prime}\right)$ is $f_{\mathrm{b}}(\varepsilon, \vec{r})$ in the $j$ th spatial bin, and the summation is over the entire energy range, the cumulative probability is

$$
\Pi_{j}\left(\varepsilon_{i}^{\prime}\right)=\sum_{k=1}^{i} P_{j}\left(\varepsilon_{k}^{\prime}\right) / \sum_{k} P_{j}\left(\varepsilon_{k}^{\prime}\right),
$$

where the summation in the numerator is over lower energies, so that $\sum_{i} \Pi_{j}\left(\varepsilon_{i}^{\prime}\right)=1$. The energy of the target electron $\varepsilon_{i}^{\prime}$ is that which satisfies

$$
\Pi_{j}\left(\varepsilon_{i-1}^{\prime}\right)<r_{7} \leqslant \Pi_{j}\left(\varepsilon_{i}^{\prime}\right) .
$$

Once the velocity of the collision partner, $\vec{v}^{\prime}$, is chosen, the impact parameter for a $90^{\circ}$ scattering, $b_{0}$, is determined as [17]

$$
b_{0}=e^{2} /\left(2 \pi \varepsilon_{0} m_{\mathrm{e}} g^{2}\right),
$$

where $m_{\mathrm{e}}$ is the mass of electron, $\vec{g}=\vec{v}_{0}-\vec{v}^{\prime}$ is the velocity between the collision partners, $g$ is the speed and $\varepsilon_{0}$ is the vacuum permittivity. If $\cos (\theta)<b_{0} / \lambda_{\mathrm{D}}$, where $\theta$ is the angle between velocity of the pseudo-electron and its collision partner and $\lambda_{\mathrm{D}}$ is the local Debye length, the collision event is ignored. Although scattering through very small angles may not be well represented by this approach, such scattering does not appreciably affect $f_{\mathrm{b}}(\varepsilon)$ at energies which determine inelastic rate coefficients. Otherwise, the probability of an e-e collision during the current time step $\Delta t$ is determined from

$$
P_{\mathrm{ee}}(g, \Delta t)=n_{j} \sigma_{\mathrm{ee}}(g) g \Delta t,
$$

where $n_{j}$ is the density of electrons in the $j$ th spatial bin obtained from the FKS, and the momentum transfer Coulomb cross section, $\sigma_{\mathrm{ee}}(g)$, is [18]

$$
\sigma_{\mathrm{ee}}(g)=4 \pi b_{0}^{2}\left[1+\ln \left(\lambda_{\mathrm{D}} / b_{0}\right)^{2}\right]^{1 / 2} .
$$

This procedure is justified if, for the conditions of interest, $P_{\text {ee }}(g, \Delta t) \ll 1$, which is the case for virtually all conditions of 
this study. The collision event takes place if $P_{\mathrm{ee}}(g, \Delta t) \geqslant r_{8}$. If a collision occurs, then a post-collision relative velocity, $\vec{g}^{\prime}$, is randomly determined such that [19]

$$
\begin{gathered}
g_{z}^{\prime}= \pm|\vec{g}| r_{9}, \quad g_{x}^{\prime}=|\vec{g}| \sqrt{1-r_{9}^{2}} \cos \left(2 \pi r_{10}\right), \\
g_{y}^{\prime}=|\vec{g}| \sqrt{1-r_{9}^{2}} \sin \left(2 \pi r_{10}\right),
\end{gathered}
$$

where $g_{z}^{\prime}$ is positive or negative if $g_{z}$ is positive or negative. The final velocity of the incident pseudoparticle, $\vec{v}_{\mathrm{f}}$, is updated with

$$
\vec{v}_{\mathrm{f}}=\vec{v}_{R}+0.5 \vec{g}^{\prime} \quad \vec{v}_{R}=0.5\left(\vec{v}^{\prime}+\vec{v}_{0}\right) .
$$

At the end of a call to the eMCS, $f_{\mathrm{b}}(\varepsilon, \vec{r})$ are used to compute electron-impact rate coefficients $k_{j}(\vec{r})$ for collision process $j$,

$$
k_{j}(\vec{r})\left(\mathrm{cm}^{3} \mathrm{~s}^{-1}\right)=\sum_{i=1} f_{\mathrm{b} i}(\vec{r}) \sigma_{j}\left(\varepsilon_{i}\right)\left(\frac{2 \varepsilon_{i}}{m_{\mathrm{e}}}\right)^{1 / 2} \Delta \varepsilon_{i} .
$$

The values of $k_{j}(\vec{r})$ are held constant in the FKS until the next call to the eMCS. The source function resulting from the rate coefficient in the fluid modules is then $S_{j}(\vec{r})\left(\mathrm{cm}^{-3} \mathrm{~s}^{-1}\right)=$ $n_{\mathrm{e}}(\vec{r}) k_{j}(\vec{r}) N_{j}(\vec{r})$, for electron density $n_{\mathrm{e}}$ and heavy particle collision partner $N_{j}$.

A similar process is followed to obtain the electron energy distributions of secondary electrons that are first emitted from surfaces and accelerated by sheaths, $f_{\mathrm{s}}(\varepsilon, \vec{r})$. Instead of seeding electrons in the bulk plasma, the flux of energetic particles (ions, photons, excited states) of type $j$ striking surfaces, $\phi_{j}(\vec{r})$ is obtained from the FKS. The total rate of secondary-electron emission, $R_{\mathrm{S}},\left(\mathrm{s}^{-1}\right)$ is obtain from

$$
R_{\mathrm{S}}=\sum_{k} R_{\mathrm{S} k}, \quad R_{\mathrm{S} k}=\sum_{j} \gamma_{j} \phi_{j}\left(\vec{r}_{k}\right) A_{k}
$$

where the summation is over species $j$ having secondaryelectron emission coefficient $\gamma_{j}$ and surface locations $k$ having surface areas $A_{k}$. A preselected number of secondary electrons, typically 25000-50000 per call to the eMCS, are then randomly launched perpendicularly to the surface with an energy of $4 \mathrm{eV}$ from spatial location $k$ in proportion to $R_{\mathrm{S} k} / R_{\mathrm{S}}$ with initial times randomly distributed in the LF rf cycle. The statistical weighting of the particle, $w$, has units of current or particles/s. Particle trajectories are then tracked and statistics collected to produce $f_{\mathrm{s}}(\varepsilon, \vec{r})$ is the same manner as for the bulk $f_{\mathrm{b}}(\varepsilon, \vec{r})$. Since the weightings $w$ of the secondary-electron pseudoparticles are particles/s, the distribution functions $f_{\mathrm{s}}(\varepsilon, \vec{r})$ are normalized,

$$
\sum_{j} F_{i j}=\sum_{i, j} f_{\mathrm{s} i j} \varepsilon_{i}^{1 / 2} \Delta \varepsilon_{i} \Delta \mathrm{V}_{\mathrm{j}}
$$

where $\Delta \mathrm{V}_{\mathrm{j}}$ is the volume of the cell at location $j$ and $f_{\mathrm{s} i j}=f_{\mathrm{s}}\left(\varepsilon_{i}\right)$. With this normalization, $f_{\mathrm{s}}(\varepsilon, \vec{r})$ has units of electrons $/ \mathrm{cm}^{3} \mathrm{eV} \mathrm{s}$.

The trajectories of pseudoparticles are followed until the particle strikes a surface or falls below a specified energy, which is typically the lowest electronic excitation threshold, $\varepsilon_{\mathrm{T}}$. At that time, the pseudoparticle is removed from the simulation. The weighting of originally emitted pseudoparticles falling below $\varepsilon_{\mathrm{T}}$ is summed into a current source, $Q_{\mathrm{e}}(\vec{r})\left(\mathrm{C} \mathrm{cm}^{-3} \mathrm{~s}^{-1}\right)$,

$$
\sum_{j^{\prime}=j, \text { neighbors }}\left[Q_{j^{\prime}} \rightarrow Q_{j^{\prime}}+\frac{q w \eta_{j j^{\prime}}}{\Delta V_{j^{\prime}}}\right],
$$

where $Q_{j}$ is the current source for location $j$ having volume $\Delta V_{j}$. Secondary electrons emitted from surfaces represent a source of electrons for the electron continuity equation in the fluid simulation. This source is provided by $Q_{\mathrm{e}}(\vec{r})$. Since this source does not also appear in a positive ion continuity equation, it appears as net charge injection in solution of Poisson's equation through the change in electron density. Secondary electrons striking surfaces are similarly summed into a rate of surface charging $Q_{\mathrm{se}}(\vec{r})\left(\mathrm{C} \mathrm{cm}^{-2} \mathrm{~s}^{-1}\right)$ which is then included in the continuity equation for surface charging in the fluid modules.

Since $f_{\mathrm{s}}(\varepsilon, \vec{r})$ is ultimately normalized to the magnitude of the secondary-electron current, $R_{\mathrm{S}}$, instead of rate coefficients being transferred back to the fluid modules, electron-impact source functions due to secondary electrons are returned to the FKS,

$$
S_{\mathrm{e} j}(\vec{r})\left(\mathrm{cm}^{-3} \mathrm{~s}^{-1}\right)=N_{j}(\vec{r}) \sum_{i=1} f_{\mathrm{s} i}(\vec{r}) \sigma_{j}\left(\varepsilon_{i}\right)\left(\frac{2 \varepsilon_{i}}{m_{\mathrm{e}}}\right)^{1 / 2} \Delta \varepsilon_{i} .
$$

The values of $S_{\mathrm{e} j}(\vec{r})$ are held constant until the next call to the eMCS.

This hybrid method of obtaining and utilizing $f_{\mathrm{b}}(\varepsilon, \vec{r})$ and $f_{\mathrm{s}}(\varepsilon, \vec{r})$ is sometimes referred to as time slicing, as a slice of time is separately addressed by each module. For any given call to the eMCS or FKS, the time integration within that module does capture transient behavior. For example, the time step in the FKS for update of species densities and between solutions of Poisson's equation is about $10^{-11} \mathrm{~s}$ with consideration of the Courant limit and resolution of the rf cycles. The time step within the eMCS is similarly small. However, the ability to represent transients truly consistently with changes in $f(\varepsilon, \vec{r})$ in the FKS, and with changes in densities within the eMCS, is determined by the frequency with which there is information exchange between the FKS and the eMCS. In this work, the eMCS is called every $0.5 \mu \mathrm{s}$, which is also the time of integration of pseudoparticle trajectories in the eMCS. With a PRF of $50 \mathrm{kHz}(20 \mu \mathrm{s})$, there are 40 updates of $f(\varepsilon)$ during one pulse period, which might be considered a lower limit of time resolution. The effective time resolution of transients is finer than that due to the finer integration within each module.

The reaction mechanisms for the $\mathrm{Ar}$ and $\mathrm{Ar} / \mathrm{CF}_{4} / \mathrm{O}_{2}$ mixtures used in this investigation are discussed in [20]. For $\mathrm{Ar}$, the species included in the model are metastable and radiative states of $\operatorname{Ar}(4 s), \operatorname{Ar}(4 p)$ and $\mathrm{Ar}^{+}$. For $\mathrm{Ar} / \mathrm{CF}_{4} / \mathrm{O}_{2}$, the additional species are $\mathrm{CF}_{4}, \mathrm{C}_{2} \mathrm{~F}_{6}, \mathrm{C}_{2} \mathrm{~F}_{4}, \mathrm{CF}_{3}, \mathrm{CF}_{2}, \mathrm{CF}, \mathrm{C}$, $\mathrm{F}, \mathrm{F}_{2}, \mathrm{CF}_{3}^{+}, \mathrm{CF}_{2}^{+}, \mathrm{CF}^{+}, \mathrm{C}^{+}, \mathrm{F}_{2}^{+}, \mathrm{F}^{+}, \mathrm{CF}_{3}^{-}, \mathrm{F}^{-}, \mathrm{O}_{2}, \mathrm{O}_{2}\left({ }^{1} \Delta\right), \mathrm{O}_{2}^{+}, \mathrm{O}$, $\mathrm{O}\left({ }^{1} \mathrm{D}\right), \mathrm{O}^{+}, \mathrm{O}^{-}, \mathrm{COF}, \mathrm{COF}_{2}, \mathrm{CO}_{2}, \mathrm{FO}, \mathrm{SiF}_{4}, \mathrm{SiF}_{3}$ and $\mathrm{SiF}_{2}$. For the operating conditions in this work, the dominant ions and neutral radicals are $\mathrm{CF}_{3}^{+}, \mathrm{CF}_{3}, \mathrm{CF}_{2}, \mathrm{CF}, \mathrm{C}, \mathrm{F}$ and $\mathrm{O}$. Vibrational excitation collisions of all molecular species are included in solving for $f(\varepsilon)$. In particular for the feedstock gases, we include $\mathrm{CF}_{4}(v 1,3), \mathrm{CF}_{4}(v 2,4)$ and $\mathrm{O}_{2}(v 1$ to $v 6)$. Threshold energies for these processes are listed in [20]. Although 


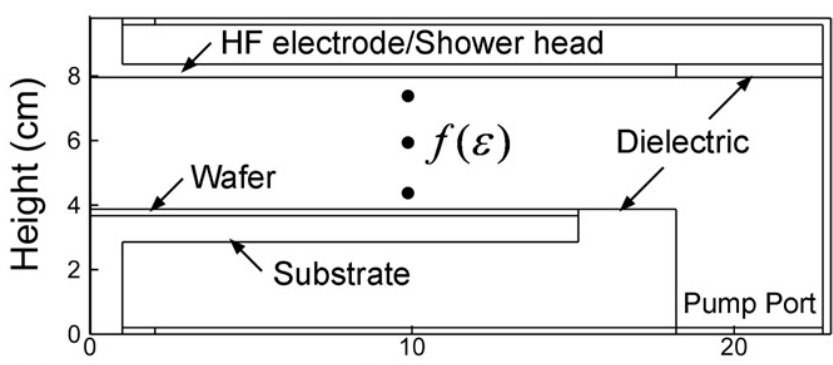

(a)

Radius (cm)

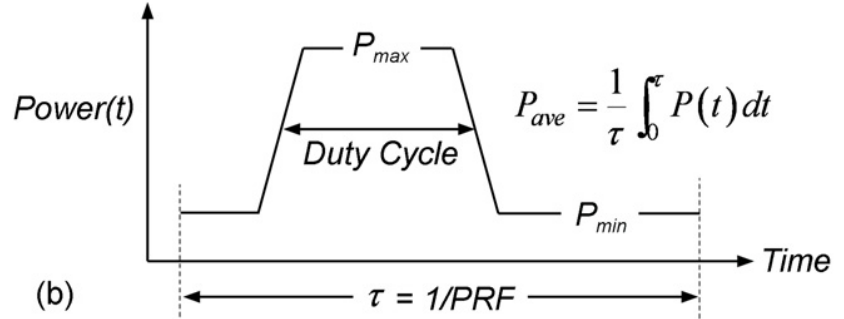

Figure 1. Operating conditions for this investigation. (a) Geometry of the DF-CCP chamber. The LF $(10 \mathrm{MHz})$ is applied on the lower electrode in $\mathrm{CW}$ mode, and the $\mathrm{HF}(40 \mathrm{MHz})$ is applied on the upper electrode in pulse mode with a few tens of $\mathrm{kHz}$ PRF. The dots show where $f(\varepsilon)$ will be plotted. (b) Pulsed operation is determined by the DC and PRF. The power is turned on during the fraction of the total period designated by the DC. PRF is how many times per second the pulse waveform repeats.

electron energy losses for exciting these vibrational states are included in the eMCS, the vibrational states are not explicitly included in the continuity equations. The consequence of this approach is that superelastic relaxation and gas heating resulting from electron and heavy particle $V-T$ collisions with these states are not captured. The secondary-electron emission coefficient for all ions is $\gamma=0.15$. The voltage is specified for each frequency and applied to the electrode. A blocking capacitor is in series with the LF electrode and a time dependent dc bias is computed based on the value of the blocking capacitor and integral of collected current. The voltages on the electrodes are adjusted to provide a specified pulsed-cycle averaged power, $\bar{P}$. This is accomplished by computing for each electrode

$$
\bar{P}=v_{\mathrm{rf}} \iint\left(V(t)\left(j(\vec{r}, t)+\varepsilon \frac{\mathrm{d} E(\vec{r}, t)}{\mathrm{d} t}\right) \mathrm{d} t \mathrm{~d} A\right),
$$

where $V$ is the voltage on the electrode, $j$ is the conduction current density to the electrode, $\varepsilon$ is the permittivity, $E$ is the electric field at the surface of the electrode and the integral is over the area of the electrode and the $\mathrm{rf}$ cycle having frequency $v_{\mathrm{rf}}$.

\section{Plasma properties of pulse powered DF-CCP sustained in Ar}

The two-dimensional, cylindrically symmetric reactor used in the model is schematically shown in figure 1 . The lower electrode serves as the substrate which is powered at a LF, $10 \mathrm{MHz}$, through a blocking capacitor $(1 \mu \mathrm{F})$. A conductive $\mathrm{Si}$ wafer $\left(\varepsilon / \varepsilon_{0}=12.0, \sigma=0.01 \Omega^{-1} \mathrm{~cm}^{-1}\right), 30 \mathrm{~cm}$ in diameter, sits in electrical contact with the substrate which is surrounded by a dielectric $\left(\varepsilon / \varepsilon_{0}=8.0, \sigma=10^{-6} \Omega^{-1} \mathrm{~cm}^{-1}\right)$. The upper electrode, $36 \mathrm{~cm}$ in diameter, is powered at a HF, $40 \mathrm{MHz}$. The HF electrode also serves as the shower head through which gas is injected at $200 \mathrm{sccm}$. The HF electrode is surrounded by the same dielectric as the LF electrode. The gap between the two electrodes is $4 \mathrm{~cm}$. All other surfaces in the reactor are grounded metal including the annular pump port. LF power is delivered in $\mathrm{CW}$ operation and $\mathrm{HF}$ power is delivered in either $\mathrm{CW}$ or pulsed format. The pulse operation was characterized by the PRF which is the number of times per second the waveform is repeated and the DC, which is the fraction of the total time of power-on stage, as shown in figure $1(b)$. The rise (or decay) time of the power-on (or -off) period is $500 \mathrm{~ns}$. The voltage on the electrodes is periodically adjusted so that the power through each electrode averaged over the pulse period is the specified amount.

The base case operating conditions are 40 mTorr of $\mathrm{Ar}$ with the $\mathrm{LF}(10 \mathrm{MHz})$ delivering $500 \mathrm{~W}$ on a $\mathrm{CW}$ basis and the $\mathrm{HF}(40 \mathrm{MHz})$ delivering an average of $500 \mathrm{~W}$ in a pulse power format. The PRF is $50 \mathrm{kHz}$ (pulse period $20 \mu \mathrm{s}$ ) and DC is $25 \%$. The PRF was varied from 50 to $250 \mathrm{kHz}$ and the DC was varied from $25 \%$ to $50 \%$.

As a point of reference, the electron density $\left(n_{\mathrm{e}}\right)$, electron temperature $\left(T_{\mathrm{e}}\right)$ and electron-impact ionization sources are shown in figure 2( $a$ ) for CW operation of both the LF and HF. The adjusted voltage amplitude is $112 \mathrm{~V}$ for $\mathrm{LF}$ and $95 \mathrm{~V}$ for $\mathrm{HF}$ for both to deliver $500 \mathrm{~W}$. The resulting dc bias is $-50 \mathrm{~V}$. The peak electron density is $9.7 \times 10^{11} \mathrm{~cm}^{-3}$ and the bulk electron temperature is $T_{\mathrm{e}} \approx 1.7 \mathrm{eV}$. $T_{\mathrm{e}}$ adjacent to the electrodes is higher $(2.0 \mathrm{eV})$ than in the bulk due to the stochastic heating produced by the oscillating sheath boundary. The electron-impact ionization sources by bulk, $S_{\mathrm{b}}$, and secondary e-beam, $S_{\mathrm{s}}$, have maximum values of $3.1 \times 10^{16} \mathrm{~cm}^{3} \mathrm{~s}^{-1}$ and $6.5 \times 10^{15} \mathrm{~cm}^{3} \mathrm{~s}^{-1}$, respectively. $S_{\mathrm{b}}$ is a factor of ten larger than $S_{\mathrm{s}}$ due to the continuous electron heating at the LF and HF sheath boundaries.

The electron energy distributions, $f(\varepsilon)$, at different heights in the reactor (heights are noted in figure 1) are shown in figure $2(b)$. Due to the relatively high electron density and so high thermal conductivity, the $f(\varepsilon)$ are essentially Maxwellian at low energies and nearly indistinguishable as a function of height. However, as expected from the trends of $T_{\mathrm{e}}$, the tails of $f(\varepsilon)$ are raised adjacent to electrodes and more so near the HF electrode due to the more efficient stochastic heating at the higher frequency [21]. To compensate for the increased tail portion of $f(\varepsilon)$, the low-energy portion decreases.

$n_{\mathrm{e}}$ and $T_{\mathrm{e}}$ are shown in figure 3 and electron-impact ionization sources are shown in figure 4 at different times during the pulse period for a pulsed discharge in Ar for the base case conditions. The $\mathrm{CW}$ amplitude of the LF to deliver $500 \mathrm{~W}$ is $206 \mathrm{~V}$. Due to the varying amount of current that is collected during the pulsed period and the finite size of the blocking capacitor $(1 \mu \mathrm{F})$ the dc bias oscillates during the pulse period, here between -124 and $-157 \mathrm{~V}$. This variation in dc bias during a pulse period has been noted in [22]. The pulsed HF voltage amplitude to deliver $500 \mathrm{~W}$ averaged over the pulsed cycle is $251 \mathrm{~V}$. At the beginning of the pulse period, 

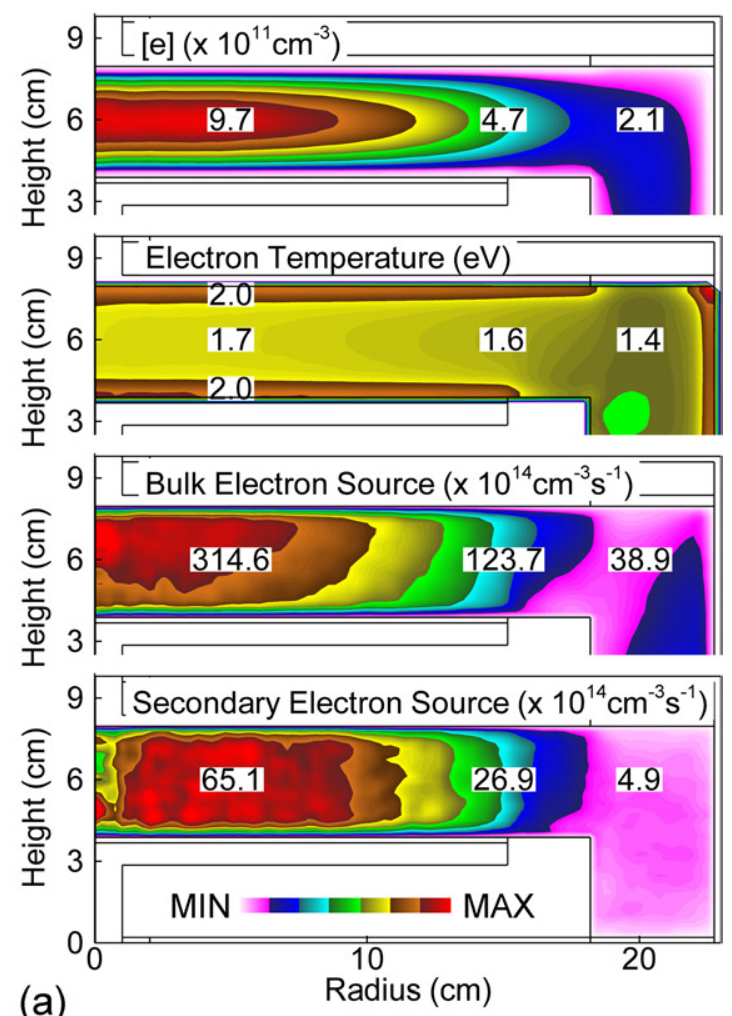

(a)

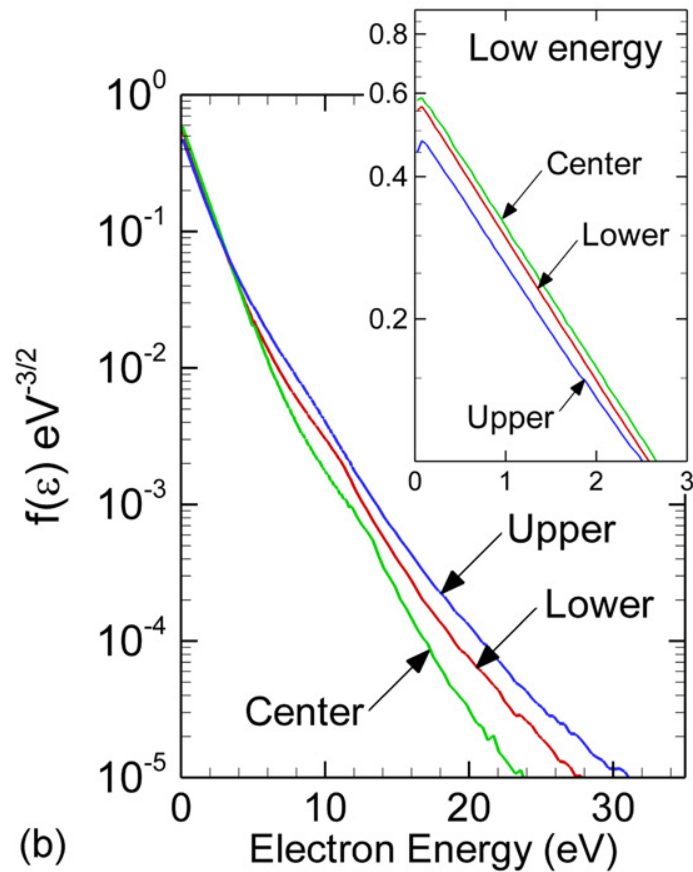

Figure 2. Plasma properties for $\mathrm{CW}$ operation in $\mathrm{Ar}$ (40 mTorr, $200 \mathrm{sccm}, 500 \mathrm{~W}$ at $10 \mathrm{MHz}, 500 \mathrm{~W}$ at $40 \mathrm{MHz}$ ). (a) Electron density, temperature and electron ionization sources by bulk electron and secondary electrons. $(b)$ Electron energy distribution at selected heights in the reactor (locations indicated in figure 1). The inset shows an enlargement of the low-energy portion of the distribution.

the maximum value of $n_{\mathrm{e}}$ is $2.5 \times 10^{11} \mathrm{~cm}^{-3}$ and the bulk $T_{\mathrm{e}} \approx 2.0 \mathrm{eV}$. There is some heating of the bulk electrons by the LF bias (to about $1.0 \mathrm{eV}$ ); however, at this high electron density, the heating by the LF is nominal. When the power is turned on, the maximum electron density increases to $3.2 \times 10^{11} \mathrm{~cm}^{-3}$.

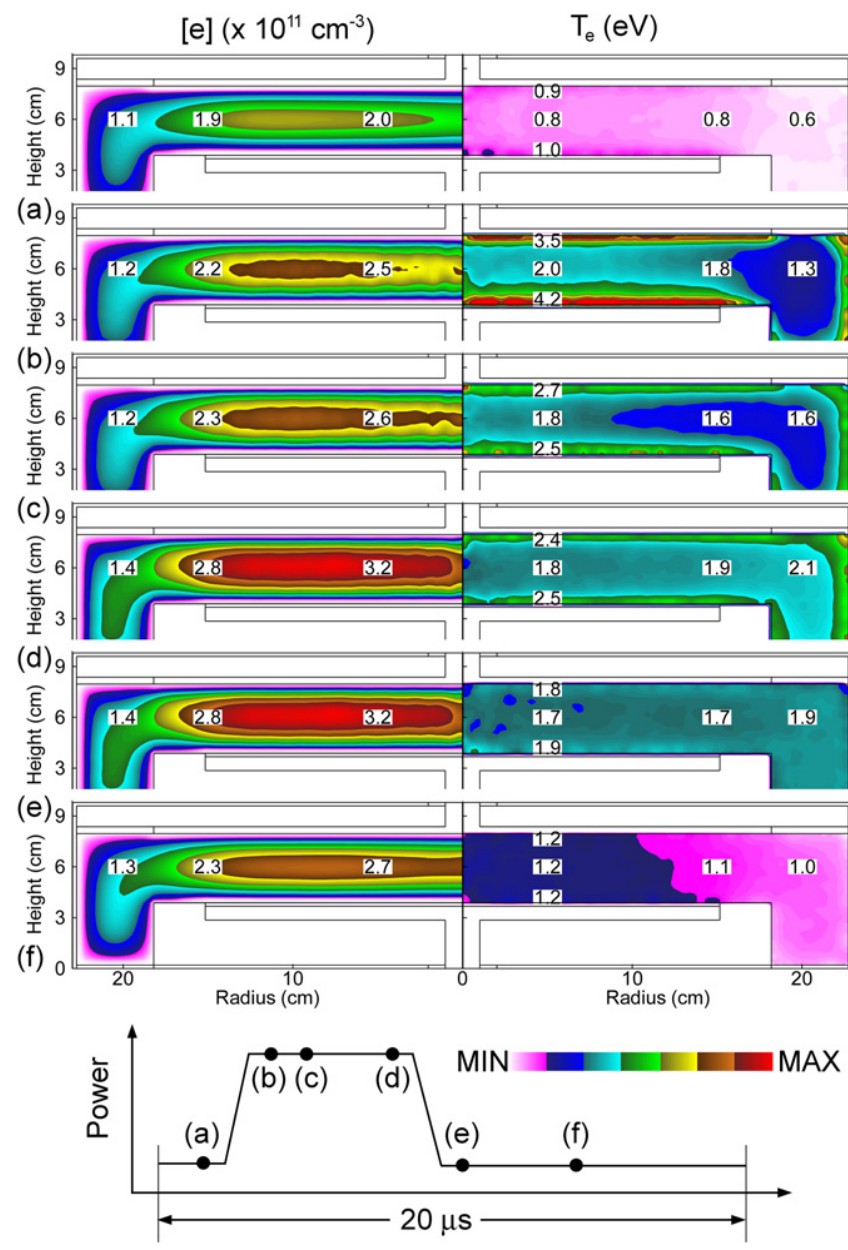

Figure 3. Electron density and temperature in Ar for the base case conditions $(40 \mathrm{mTorr}, 200 \mathrm{sccm}, 500 \mathrm{~W}$ at $10 \mathrm{MHz} \mathrm{CW}, 500 \mathrm{~W}$ at $40 \mathrm{MHz}$ in pulse mode- $50 \mathrm{kHz}$ PRF with $25 \%$ DC) at different times during the pulsed cycle (as indicated in the lower figure). The electron density does not change significantly over the pulse period, whereas the electron temperature shows instantaneous changes as the power toggles on and off, especially near the sheaths due to enhanced stochastic heating.

The more efficient stochastic electron heating by the $\mathrm{HF}$ raises $T_{\mathrm{e}}$ to $2.0 \mathrm{eV}$ in the bulk and to $4.2 \mathrm{eV}$ in the sheaths. $E / N$ (electric field/gas number density, $1 \mathrm{Td}=10^{-17} \mathrm{~V} \mathrm{~cm}^{2}$ ) near the HF sheath increases from $500 \mathrm{Td}$ to $2000 \mathrm{Td}$ during the pulse power rise time before settling to $1400 \mathrm{Td}$ during the rest of the power-on stage. With $T_{\mathrm{e}}=(2 / 3)\langle\varepsilon\rangle$ being proportional to the distribution average energy, its value is heavily weighted toward the more numerous low-energy bulk electrons. Due to the non-Maxwellian nature of $f_{\mathrm{b}}(\vec{r})$ at high energies, the dynamics of $T_{\mathrm{e}}$ are a poor measure of ionization rates. (See the discussion below.) When the HF power is turned off after a $25 \%$ DC (5 $\mu \mathrm{s}$ in this case), $T_{\mathrm{e}}$ falls to the off-period value of $1.2 \mathrm{eV}$ in about $8.5 \mu \mathrm{s}$. The PRF $(50 \mathrm{kHz})$ is high enough that the plasma density does not significantly change over the pulse period.

The electron-impact ionization sources by bulk electrons, $S_{\mathrm{b}}$, and secondary beam electrons, $S_{\mathrm{s}}$, are shown in figure $4 . S_{\mathrm{s}}$ has a continuous background value of about $5 \times 10^{14} \mathrm{~cm}^{3} \mathrm{~s}^{-1}$ due to continuous secondary-electron emission from the LF electrode. As the pulse power is toggled on and off, the ion 


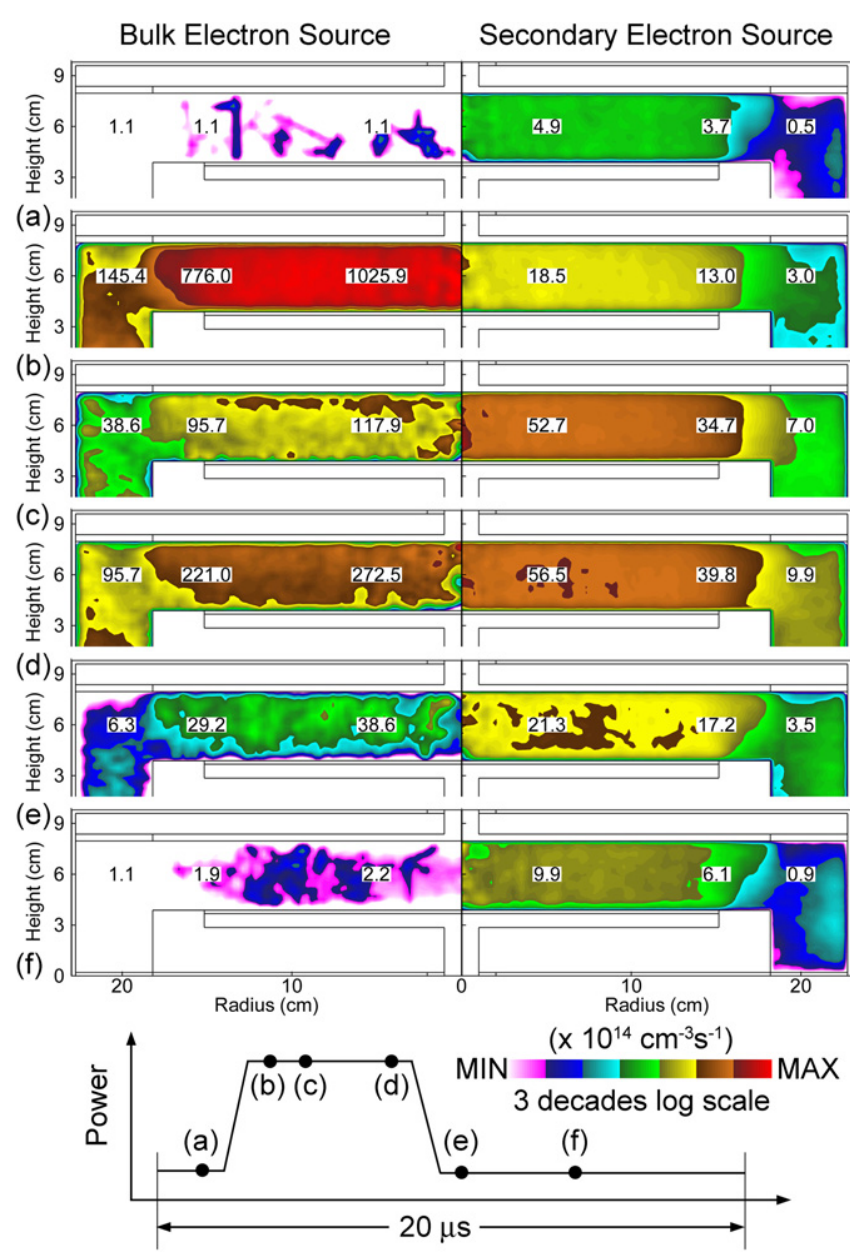

Figure 4. Ionization source by (left) bulk electrons and (right) secondary electrons in Ar for the base case conditions (40 mTorr, $500 \mathrm{~W}$ at $10 \mathrm{MHz} \mathrm{CW}, 500 \mathrm{~W}$ at $40 \mathrm{MHz}$ in pulse mode $-50 \mathrm{kHz}$ PRF with $25 \%$ DC) at different times during the pulsed cycle (as indicated in the lower figure).

density and so ion flux to surfaces do not significantly change, so this source of ionization is fairly constant. With an inelastic mean-free-path of $\approx 4 \mathrm{~cm}$ at $300 \mathrm{eV}$, the secondary electrons accelerated in the sheath cross the gap and produce a fairly spatially uniform ionization source. The delay in the peak of the ionization source is due, in part, to a time lag in increasing the secondary-electron yield due to transport of ions across the sheath from the bulk plasma.

Note that $S_{\mathrm{s}}$ increases during the pulse on period. This is due to a small decrease in the dc bias (becoming less negative) which reduces the energy of the secondary electrons which also decreases the energy relaxation length. More ionization occurs in the gap with there being less likelihood for secondary electrons to be collected by the opposite electrode. (Since during the power-off period, the HF electrode is held at ground and there is approximately a $-150 \mathrm{~V} \mathrm{dc}$ bias on the substrate, the majority of secondary electrons that cross the gap are collected by the HF electrode.) At the other extreme, there are also increasing contributions to $S_{\mathrm{s}}$ by secondary electrons emitted from the now powered HF electrode.

During the power-off period $S_{\mathrm{b}}$ is not important. Prior to turning the HF power on, there are statistically only a few electrons produced by stochastic heating by the LF sheath that have high enough energy to produce significant ionization. During the power-on portion of the cycle the additional ionization by sheath accelerated secondary electrons from the HF electrode increases $S_{\mathrm{s}}$ to $5 \times 10^{15} \mathrm{~cm}^{3} \mathrm{~s}^{-1}$, a factor of nearly ten greater than that from the LF alone. Coincident to the increase in $T_{\mathrm{e}}$ during the pulse-on period is an increase in $S_{\mathrm{b}}$, to $10^{17} \mathrm{~cm}^{3} \mathrm{~s}^{-1}$, so that bulk electrons dominate ionization. The decrease in $S_{\mathrm{b}}$ to background levels occurs in about $8.5 \mu \mathrm{s}$ after the HF power is terminated.

$f(\varepsilon)$ have dynamic behavior during the pulse period. For example, $f(\varepsilon)$ are shown in figure 5 adjacent to the HF sheath, mid-gap and adjacent to the LF sheath at different times during the pulse period. (See figure 1 for these locations.) The lowenergy component $(\varepsilon<2-3 \mathrm{eV})$ varies little during the pulse period and appears Maxwellian-like, which explains the small variation in $T_{\mathrm{e}}$ during the pulse period. (This small variation in $T_{\mathrm{e}}$ cannot explain the large change in ionization rates.) This nearly invariant part of $f(\varepsilon)$ is largely due to the thermalizing influence of electron-electron collisions. The tail of the $f(\varepsilon)$ raises and lowers nearly coincidently with the application and termination of the HF power. At its maximum extent, the tail of $f(\varepsilon)$ reaches to energies in excess of $60 \mathrm{eV}$ with only a nominal change in the low-energy portion of $f(\varepsilon)$. Although there is a HF component oscillation at the LF sheath, the amplitude of this oscillation is smaller than at the HF sheath, and the tail of $f(\varepsilon)$ extends to only $50 \mathrm{eV}$. In the middle of the gap, the extension is to $40 \mathrm{eV}$.

The pulsed period-averaged (PPA) $f(\varepsilon)$ compared with $\mathrm{CW}$ operation for the same average powers at different heights in the reactor are also shown in figure 5. The PPA $f(\varepsilon)$ adjacent to the HF electrode appear more bi-Maxwellian than with $\mathrm{CW}$ power due to the rapid and enhanced electron heating from the HF power during the power-on stage and the rapid cooling during the power-off stage. Although the PPA and CW $f(\varepsilon)$ do not show dramatic differences, the temporal dynamics of $f(\varepsilon)$ have the tail of the distribution extending to significantly higher energies than the $\mathrm{CW}$ case. This extension produces instantaneous sources for inelastic collision processes with high threshold energies that are significantly greater than either the PPA or CW distribution functions.

\section{Plasma properties in $\mathrm{Ar} / \mathrm{CF}_{4} / \mathrm{O}_{2}$}

There is an interest in plasma materials processing, and plasma etching in particular, to have additional control over the production of radicals and ions to the substrate. In this section we discuss results from the model for a DF-CCP sustained in a $\mathrm{Ar} / \mathrm{CF}_{4} / \mathrm{O}_{2}=75 / 20 / 5$ gas mixture at 40 mTorr to explore such control strategies. With this plasma being sustained in a molecular and electronegative gas mixture, there are additional volumetric electron loss processes-dissociative attachment and dissociative recombination. Although the rate coefficient for dissociative recombination scales with $T_{\mathrm{e}}^{-0.5}$ and so increases with decreasing $E / N$ and average energy, the dissociative attachment cross sections for $\mathrm{CF}_{4}$ and $\mathrm{O}_{2}$ have threshold energies of 3.0 and $5.0 \mathrm{eV}$ respectively $[23,24]$. As a result, the rate coefficient for attachment processes increase 

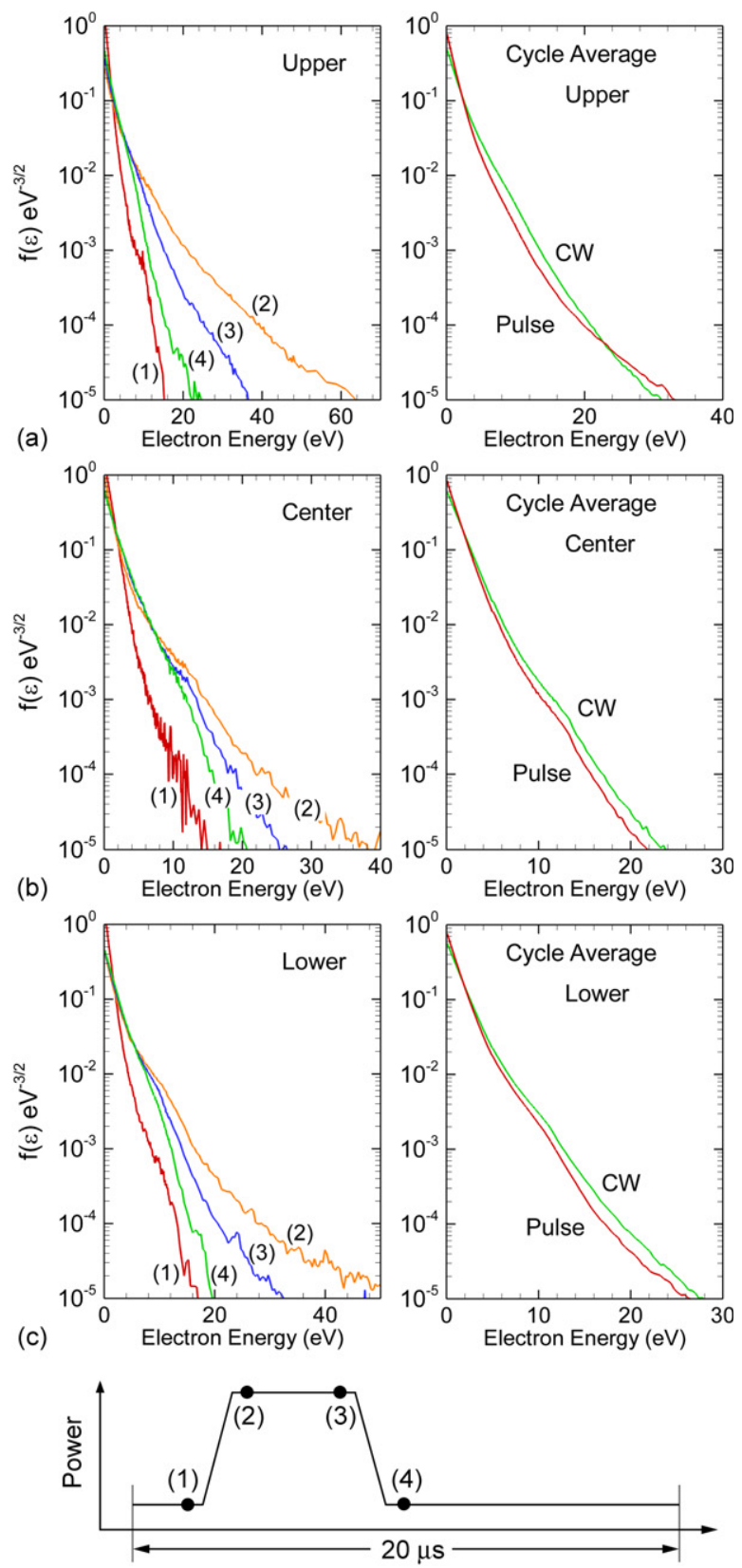

Figure 5. Electron energy distribution functions in Ar for the base case conditions ( $40 \mathrm{mTorr}, 500 \mathrm{~W}$ at $10 \mathrm{MHz} \mathrm{CW}, 500 \mathrm{~W}$ at $40 \mathrm{MHz}$ in pulse mode $-50 \mathrm{kHz}$ PRF with $25 \% \mathrm{DC}$ ) at different times during the pulse cycle (as indicated in the lower figure) for $(a)$ near the HF sheath, $(b)$ in the bulk plasma and $(c)$ near the LF sheath (locations indicated in figure 1). Comparisons of $f(\varepsilon)$ averaged over the pulse period and with $\mathrm{CW}$ excitation are in the right column. The enhanced tail of $f(\varepsilon)$ is most prominent at the leading edge of the pulse-on period.

with increasing $E / N$ up to about $100 \mathrm{Td}$. This markedly differs from thermal attaching gases with as $\mathrm{Cl}_{2}$ and $\mathrm{F}_{2}$ whose cross sections for dissociative attachment peak at energies of $<0.1 \mathrm{eV}$ and so their rate coefficients for electron loss generally decrease with increasing $E / N$ in the same manner as dissociative recombination.

As a point of reference, $n_{\mathrm{e}}, T_{\mathrm{e}}, S_{\mathrm{b}}$ and $S_{\mathrm{s}}$ are shown in figure 6 for $\mathrm{CW}$ operation for both LF and HF. Results are shown for secondary-electron emission coefficients by ion

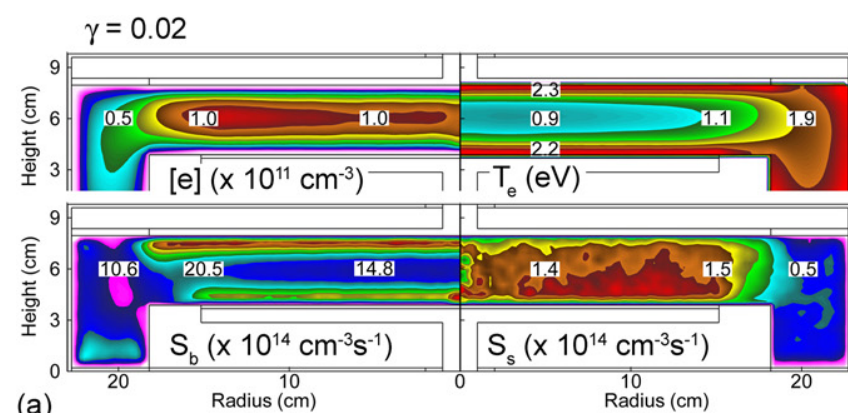

(a) $\gamma=0.10$

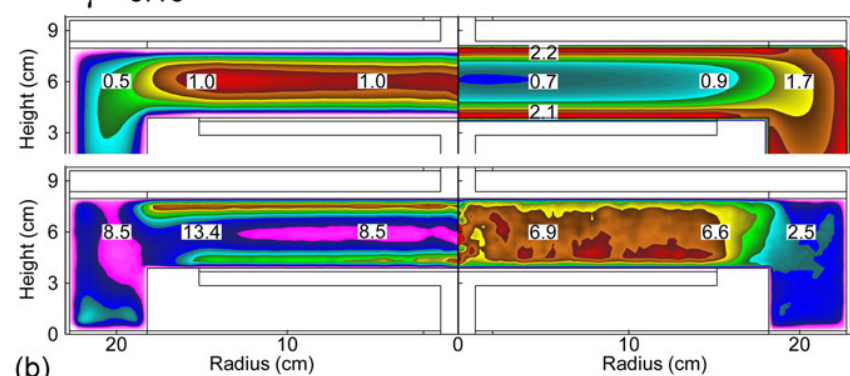

(b)
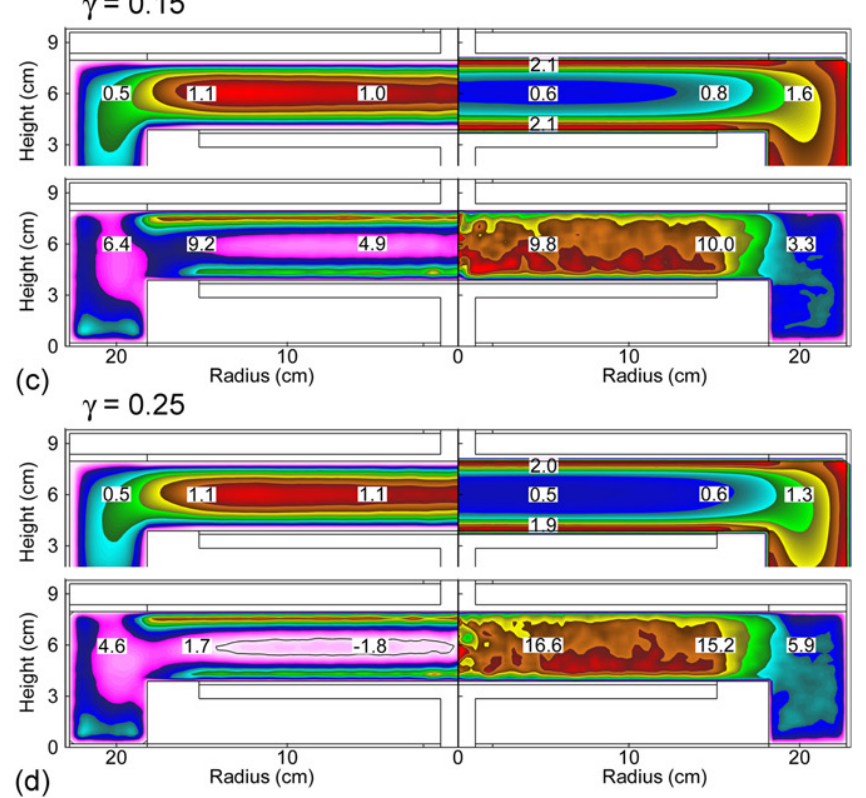

(d)

Radius $(\mathrm{cm})$

MAX

Figure 6. Electron density, temperature and electron ionization sources by bulk electron and secondary electrons in $\mathrm{Ar} / \mathrm{CF}_{4} / \mathrm{O}_{2}=75 / 20 / 5$ for $\mathrm{CW}$ excitation with different secondary-electron emission coefficients, $\gamma$. (a) 0.02, (b) 0.10, (c) 0.15 and $(d) 0.25$.

impact of $\gamma=0.02,0.10,0.15$ and 0.25 . With $\gamma=0.02$, the voltage amplitudes are $\mathrm{LF}=256 \mathrm{~V}$ and $\mathrm{HF}=155 \mathrm{~V}$ with a dc bias of $-71 \mathrm{~V}$. With $\gamma=0.15, \mathrm{LF}=259 \mathrm{~V}, \mathrm{HF}=149 \mathrm{~V}$ and $V_{\mathrm{dc}}=-75 \mathrm{~V}$. The peak electron densities are $1.0 \times 10^{11} \mathrm{~cm}^{-3}$ for $\gamma=0.02$ and 0.15 , and $T_{\mathrm{e}} \approx 0.9 \mathrm{eV}$ and $0.6 \mathrm{eV}$ for $\gamma=0.02$ and 0.15 . $T_{\mathrm{e}}$ is significantly higher adjacent to the electrodes $(2.3 \mathrm{eV})$ than in the bulk due to the stochastic heating by the oscillating sheath boundary. The larger disparity between $T_{\mathrm{e}}$ near the sheath and in the bulk compared with Ar discharge results from the shorter energy relaxation length in the molecular gas mixture. The estimated energy relaxation length in $\mathrm{Ar} / \mathrm{CF}_{4} / \mathrm{O}_{2}=75 / 20 / 5$ at a few eV is only $10 \%$ 
that in pure argon due to low threshold energy vibrational and electronic excitation collisions with $\mathrm{CF}_{4}$ and $\mathrm{O}_{2}$. Note that the larger bulk electron-impact ionization occurs adjacent to the HF electrode due to the more efficient heating by the HF sheath. The maximum secondary electron-impact ionization source leans toward the LF electrode due to the dc bias on the substrate which provides a higher average secondary energy.

While varying the secondary emission coefficient $\gamma$, the power delivered by the LF and HF remains constant. Since only a small fraction of the power deposition is by acceleration of secondary electrons, the electron (and ion) densities do not significantly change, moderately increasing with increasing $\gamma$. (Other methods of power deposition-Joule heating, stochastic and ion acceleration - are proportional to the electron or ion density.) The small increase in electron density with increasing $\gamma$ reflects the decrease in voltage amplitudes. What does change with the variation of $\gamma$ is the apportioning of ionization between bulk electrons, $S_{\mathrm{b}}$, and secondary electrons, $S_{\mathrm{s}}$. With increasing $\gamma, S_{\mathrm{s}}$ increases due to the larger flux of secondary electrons (in spite of the small decrease in sheath voltages). $S_{\mathrm{b}}$ decreases with increasing $\gamma$ to net negative values (more attachment and recombination than ionization) with $\gamma=0.25$.

The values of $n_{\mathrm{e}}, T_{\mathrm{e}}, S_{\mathrm{b}}$ and $S_{\mathrm{s}}$ are ultimately determined by a balance between electron sources by ionization (or injection) and losses (by attachment, recombination and diffusion) that provides the current that delivers the desired power. Since $S_{\mathrm{s}}$ increases by virtue of the larger $\gamma$ the plasma responds by allowing more electron loss, which is achieved by lowering $T_{\mathrm{e}}$ and $S_{\mathrm{b}}$. This is, in fact, the principle behind externally sustained plasmas, such as electron beam sustained discharges (EBSDs) [25]. In these devices, $T_{\mathrm{e}}$ in the bulk plasma is controlled by the power deposition from the electron beam. If the externally supplied ionization provides the majority of the ionization, the applied electric fields which heat electrons and determines $T_{\mathrm{e}}$ can be lower. For example, in EBSD excited $\mathrm{CO}(\mathrm{v})$ and $\mathrm{CO}_{2}(\mathrm{v})$ lasers, $T_{\mathrm{e}}$ is lowered so that rates of vibrational excitation are optimized [26, 27]. In our system, with $\gamma=0.02$, the ionization sources from $S_{\mathrm{s}}$ are insufficient to offset electron losses, and so $S_{\mathrm{b}}$ must be positive to deliver the desired power. With $\gamma=0.25$, the ionization sources $S_{\mathrm{s}}$ exceed that which is required to deliver the desired power, and so $S_{\mathrm{b}}$ is negative to compensate. So similar to an EBSD, varying $\gamma$ provides a means to control the bulk plasma properties. As an aside, another feature of relying on $S_{\mathrm{s}}$ for ionization is that the plasma is more uniform since the energy relaxation distance of the higher energy electrons is greater than for bulk electrons.

The $f(\varepsilon)$ near the HF sheath and in the center of the gap for $\gamma=0.02,0.10,0.15$ and 0.25 are shown in figure 7 . Near the HF sheath where electron transport is dominated by stochastic heating and which is little affected by changes in $\gamma, f(\varepsilon)$ are essentially unchanged with $\gamma$. In the center of the plasma where collisional Joule heating is more important in determining $f(\varepsilon)$, the tail of the distribution rises with decreasing $\gamma$ and the low-energy portion of the distribution increases with increasing $\gamma$.

In the steady state and in the absence of external ionization sources, the self-sustaining $E / N$ or $T_{\mathrm{e}}$ (that is,
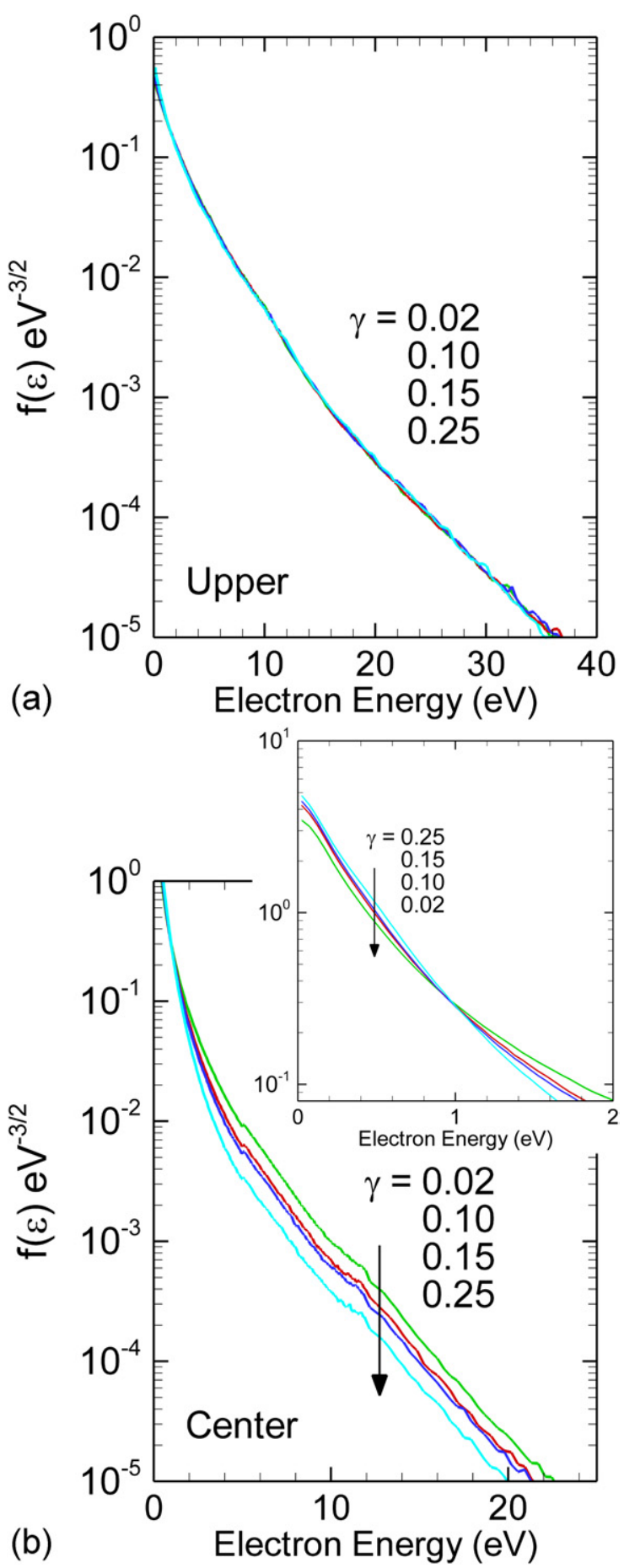

Figure 7. Electron energy distributions in $\mathrm{Ar} / \mathrm{CF}_{4} / \mathrm{O}_{2}$ with $\mathrm{CW}$ excitation for different secondary-electron emission coefficients, $\gamma$ for base case conditions. (a) Near the HF sheath and $(b)$ at the center of the plasma. With increasing rates of ionization by secondary electrons with increasing $\gamma$, the tail of the $f(\varepsilon)$ decreases.

shape of $f(\varepsilon)$ ) occurs where the volume-averaged ionization sources are balanced by losses by diffusion, attachment and recombination. In gases where volumetric losses uniformly decrease with increasing $E / N$ or $T_{\mathrm{e}}$ while ionization sources uniformly increase with increasing $E / N$ or $T_{\mathrm{e}}$, the selfsustaining value is well defined-it is the value of $E / N$ or $T_{\mathrm{e}}$ where these two curves having slopes of different sign 
intersect. In the case where both volumetric losses and ionization increase over a range of increasing $E / N$ or $T_{\mathrm{e}}$, the self-sustaining values are less clear and may, in fact, have multiple values.

In EBSDs, an external source (the injected electron beam) provides a source of ionization which is independent of the local $E / N$ or $T_{\mathrm{e}}$ (shape of $f(\varepsilon)$ ) [28]. By varying the magnitude of the external ionization provided by the electron beam by changing the beam voltage or current, the value of $E / N$ or $T_{\mathrm{e}}$ that balances ionization and losses can be tuned. This is the effect that we see in the $\mathrm{Ar} / \mathrm{CF}_{4} / \mathrm{O}_{2}$ gas mixture. If $\gamma$ is large, the 'external' source of ionization provided by ionization by secondary electrons is large enough to sustain the plasma and, in fact, may be larger than what is required to deliver the desired power. In those cases, the net ionization by bulk electrons is negative, and the tail of $f(\varepsilon)$ is depressed. (That is, the impedance of the plasma is small, producing a small $E / N$ and so reduced collisional heating.) If $\gamma$ is small, the 'external' source of ionization provided by secondary electrons cannot sustain the plasma, and so the tail of $f(\varepsilon)$ is raised to provide the required ionization. (That is, the impedance of the plasma is large, producing a large $E / N$ and increased collisional heating.) To some degree, the bulk $f(\varepsilon)$ can be tuned by varying the amount of external ionization provided by the secondary electrons by varying the secondaryelectron emission coefficient.

The conditions for pulsed operation of the $\mathrm{Ar} / \mathrm{CF}_{4} / \mathrm{O}_{2}$ mixture are the same as for the base case in argon (40 mTorr, $200 \mathrm{sccm}$, LF delivering $500 \mathrm{~W}$ at $10 \mathrm{MHz}, \mathrm{HF}$ delivering $500 \mathrm{~W}$ at $40 \mathrm{MHz}, \mathrm{PRF}=50 \mathrm{kHz}, \mathrm{DC}=25 \%$ ). The amplitude of the LF to deliver $500 \mathrm{~W}$ is $202 \mathrm{~V}$ with a dc bias varying between -54 and $-93 \mathrm{~V}$ during the pulse period. $n_{\mathrm{e}}$ and $T_{\mathrm{e}}$ at selected times over the pulse period are shown in figure 8 with $\gamma=0.15$. $E / N$ near the HF sheath is shown in figure 9. Electron-impact ionization sources by bulk and secondary electrons are shown in figure 10. The dynamic range of change in these properties is greater than for the pure argon case due to the higher collisionality and higher rate of volumetric electron loss due to recombination and attachment. Prior to the application of the pulse power, $T_{\mathrm{e}}$ in the bulk plasma is as low as $0.4 \mathrm{eV}$ and only $0.8 \mathrm{eV}$ adjacent to the sheaths. This value of $T_{\mathrm{e}}$ is enabled, in part, by the continuous background value of $S_{\mathrm{s}}$ due to the LF bias. When the HF power is turned on, $T_{\mathrm{e}}$ increases within $0.5 \mu \mathrm{s}$ from 0.8 to $4.7 \mathrm{eV}$ adjacent to the HF sheath, and to $1.9 \mathrm{eV}$ in the bulk plasma. This is accompanied by an increase in the maximum $n_{\mathrm{e}}$ from $1.3 \times 10^{11}$ to $1.7 \times 10^{11} \mathrm{~cm}^{-3}$. These values of $T_{\mathrm{e}}$ relax during the power-on period, to $2.1 \mathrm{eV}$ adjacent to the sheaths and $0.8 \mathrm{eV}$ in the bulk, before returning to their pre-pulse value at the termination of the HF power. The increase in $T_{\mathrm{e}}$ at the beginning of the power-on stage is due to an overshoot of $E / N$ above the quasi-steady state during the pulse. Although not as severe, such overshoot is common in pulsed ICPs [29]. In pulsed ICPs, the overshoot is due to the larger power dissipation into a smaller density of surviving electrons at the end of the afterglow. At the boundary of HF sheath, the $E / N$ changes from $200 \mathrm{Td}$ to $2500 \mathrm{Td}$ during the pulse rise before settling down to $1400 \mathrm{Td}$ for the power-on stage, as shown in figure 9.

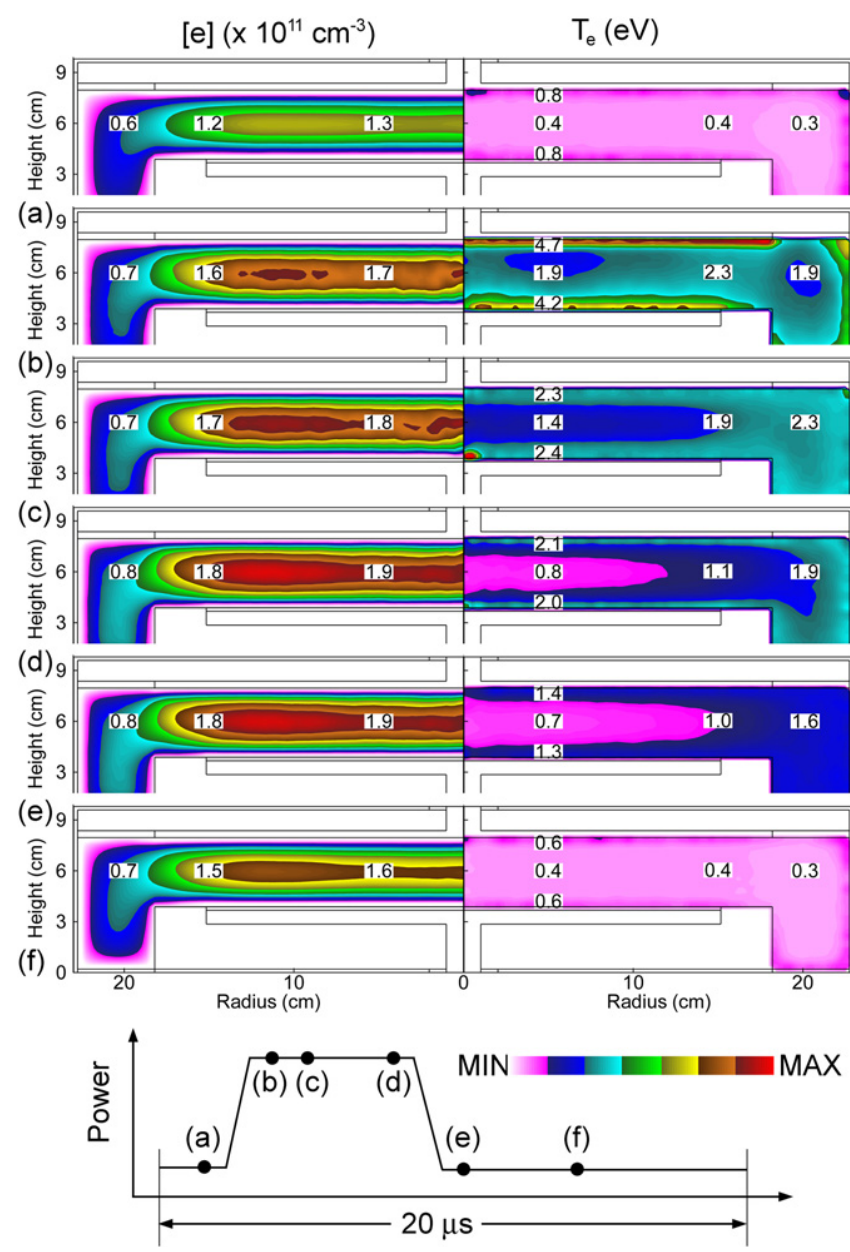

Figure 8. Plasma properties with pulsed excitation in $\mathrm{Ar} / \mathrm{CF}_{4} / \mathrm{O}_{2}=75 / 20 / 5$ - (left) electron density and (right) electron temperature for the base case conditions $(40 \mathrm{mTorr}, 500 \mathrm{~W}$ at $10 \mathrm{MHz} \mathrm{CW}$ and $500 \mathrm{~W}$ at $40 \mathrm{MHz}$ in pulse mode $-50 \mathrm{kHz}$ PRF with $25 \%$ DC) at different times during the pulsed cycle (as indicated in the lower figure).

As in the CW cases for high values of $\gamma$, the net ionization by bulk electrons, $S_{\mathrm{b}}$, averaged over the pulse period is negative. During the power-off period, $S_{\mathrm{b}}$ is as large (negative) as $-3.5 \times 10^{15} \mathrm{~cm}^{3} \mathrm{~s}^{-1}$, which is primarily due to attachment (as opposed to recombination). Although the cross section for dissociative electron attachment to $\mathrm{CF}_{4}$ and $\mathrm{O}_{2}$ with few eV electrons is 3 orders of magnitude smaller than the cross section for the recombination of $\mathrm{CF}_{3}^{+}$and $\mathrm{O}_{2}^{+}$, the number density of $\mathrm{CF}_{4}$ and $\mathrm{O}_{2}$ is 4 to 6 orders larger than the $\mathrm{CF}_{3}^{+}$and $\mathrm{O}_{2}^{+}$. At the leading edge of the pulsed power, an increase in $T_{\mathrm{e}}$ produces a momentary positive increase in $S_{\mathrm{b}}$ to $9 \times 10^{16} \mathrm{~cm}^{3} \mathrm{~s}^{-1}$ during the overshoot in $E / N$ and remains net positive during the rest of the power-on pulse. During the pulse-off period, $S_{\mathrm{b}}$ is negative. The ionization balance is provided by the secondary electrons. As with the Ar discharge, there is a background $S_{\mathrm{s}}$ due to the LF bias of $3 \times 10^{14} \mathrm{~cm}^{3} \mathrm{~s}^{-1}$. This ionization source is not large enough to balance attachment on a CW basis - the increase in $S_{\mathrm{s}}$ during the pulse-on period to $2 \times 10^{15} \mathrm{~cm}^{3} \mathrm{~s}^{-1}$ coupled with the momentary increase in $S_{\mathrm{b}}$ provides the pulse-averaged ionization balance. Due to the resonant dissociative attachment cross sections, there is 


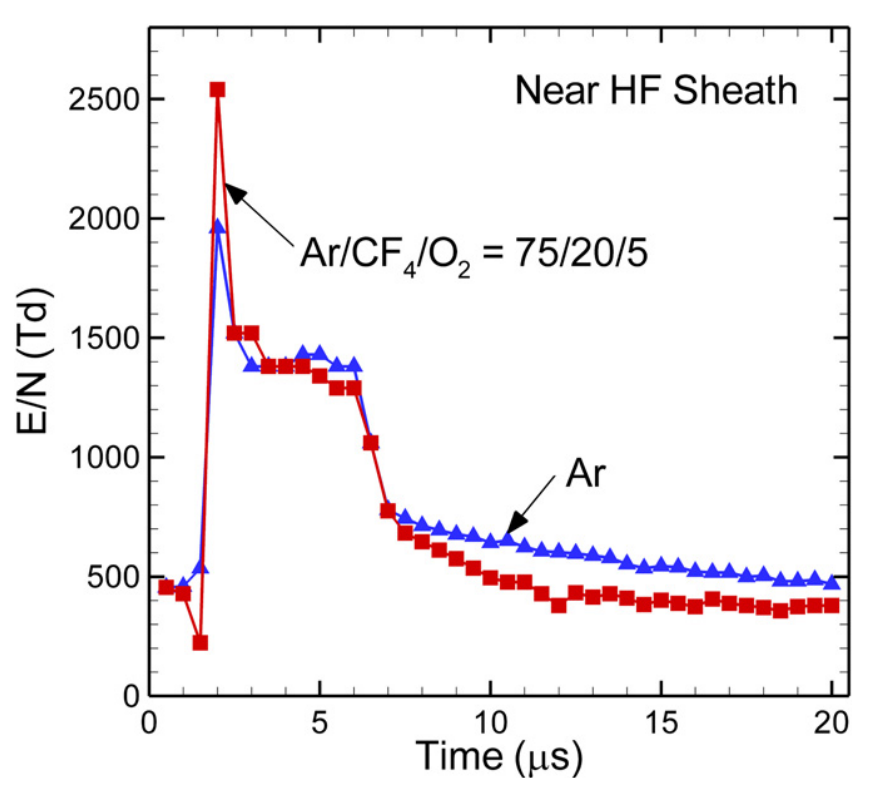

Figure 9. $E / N$ over the pulsed cycle with $\mathrm{Ar}$ and $\mathrm{Ar} / \mathrm{CF}_{4} / \mathrm{O}_{2}=75 / 20 / 5$ for base case conditions $(40 \mathrm{mTorr}, 500 \mathrm{~W}$ at $10 \mathrm{MHz} \mathrm{CW}$ and $500 \mathrm{~W}$ at $40 \mathrm{MHz}$ in pulse mode $-50 \mathrm{kHz}$ PRF with $25 \%$ DC). The overshoot is more severe in the $\mathrm{Ar} / \mathrm{CF}_{4} / \mathrm{O}_{2}$ mixture due to the lower electron density at the beginning of the pulse.

essentially no volumetric electron loss for energies greater than $15-20 \mathrm{eV}$. Since the majority of the secondary-electron energies considerably exceed $15-20 \mathrm{eV}$, they make a negligible contribution to volumetric losses.

$f(\varepsilon)$ near the sheaths and in the bulk plasmas (locations shown in figure $1(a)$ ) at different times during the pulse period are shown in figure 11 for the base case conditions. As the pulsed power is toggled on and off, the high-energy electron population in the tail of the $f(\varepsilon)$ is modulated to high and low values. This modulation is more extreme in this gas mixture compared with the pure argon case. The tail of $f(\varepsilon)$ at the $\mathrm{HF}$ sheath extends to over $120 \mathrm{eV}$ at the leading edge of the power-on period due to the overshoot of $E / N$ at the leading edge of the pulse-on period above the steady-state value. The $f(\varepsilon)$ then adjusts quickly to the power-on value after the local enhancement in $E / N$ diminishes. The enhancement in the tail of $f(\varepsilon)$ at the LF sheath is to $60 \mathrm{eV}$, and in the bulk plasma to $50 \mathrm{eV}$. The rate at which the high-energy tail $f(\varepsilon)$ collisionally relaxes is greater than in the argon case due to the lower energy inelastic thresholds in this gas mixture. The PPA $f(\varepsilon)$ are also compared with the CW $f(\varepsilon)$ in figure 11. The disparity between the PPA and CW distributions is most acute near the HF sheath where the PPA $f(\varepsilon)$ is both more thermal (larger low-energy component) and has a more extensive high-energy tail. This results from thermalization during the power-off period (enhancing the low-energy component) and stochastic heating at the leading edge of the power-on period (enhancing the high-energy tail).

The distribution and mole fraction-averaged rate coefficient for all electron-impact ionization processes and for all electron loss processes due to bulk electrons in the middle of the reactor are shown in figure 12 for the base case conditions in pure argon and $\mathrm{Ar} / \mathrm{CF}_{4} / \mathrm{O}_{2}$. In Ar, the

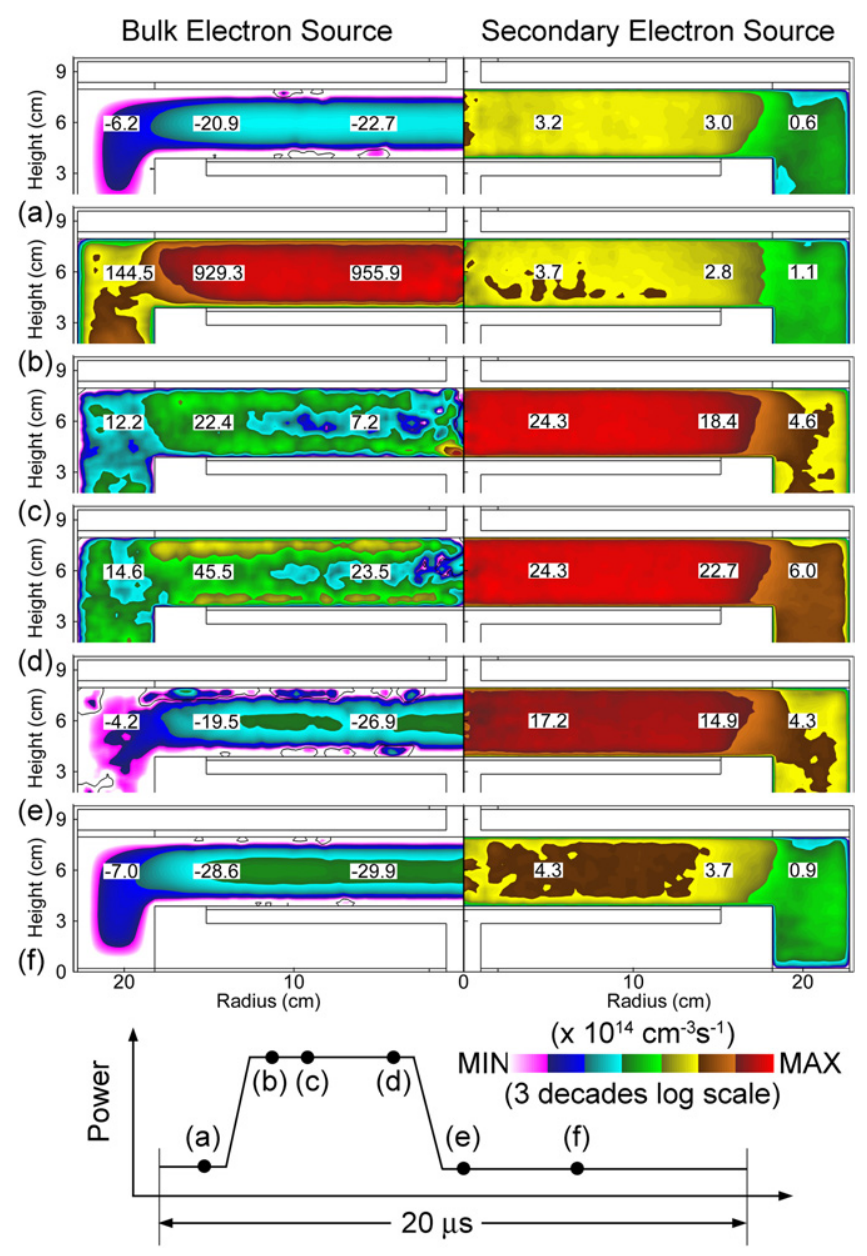

Figure 10. Ionization source by (left) bulk electrons and (right) secondary electrons in $\mathrm{Ar} / \mathrm{CF}_{4} / \mathrm{O}_{2}=75 / 20 / 5$ for the base case conditions (40 mTorr, $500 \mathrm{~W}$ at $10 \mathrm{MHz} \mathrm{CW}$ and $500 \mathrm{~W}$ at $40 \mathrm{MHz}$ in pulse mode $-50 \mathrm{kHz}$ PRF with $25 \%$ DC) at different times during the pulsed cycle (as indicated in the lower figure). The ionization source by bulk electrons is largest at the beginning of the power-on stage due to the expansion of the sheath with the application of voltage.

only measurable volumetric loss is a radiative recombination $\left(k \approx 10^{-13} / T_{\mathrm{e}}(\mathrm{eV})^{0.5} \mathrm{~cm}^{3} \mathrm{~s}^{-1}\right.$ ), and whose contribution is negligible for these conditions. The modulation in ionization rate coefficient is a factor of 40 during the pulse cycle (nearly 140 when considering the overshoot at the beginning of the power-on). The finite ionization rate coefficient during the power-off period results from the continuous heating from LF power on the substrate. Although the superelastic relaxation of Ar metastable states produces some amount of electron heating, the contribution of the superelastic relaxation during the pulse-off period is small and equivalent to $E / N=0.65 \mathrm{Td}$. In $\mathrm{Ar} / \mathrm{CF}_{4} / \mathrm{O}_{2}$ mixtures, the average bulk rate coefficient for ionization increases by $\approx 100$ during the pulse period (nearly 2000 when considering the overshoot at the beginning of the power-on). In this mixture, there is significant collisional loss of electrons, which results in a net collisional loss during the power-off period which exceeds ionization. The rapid quenching of Ar metastable states reduces significant sources of superelastic heating during the power-off period. Note that the electron loss rate coefficient increases during the power-on 

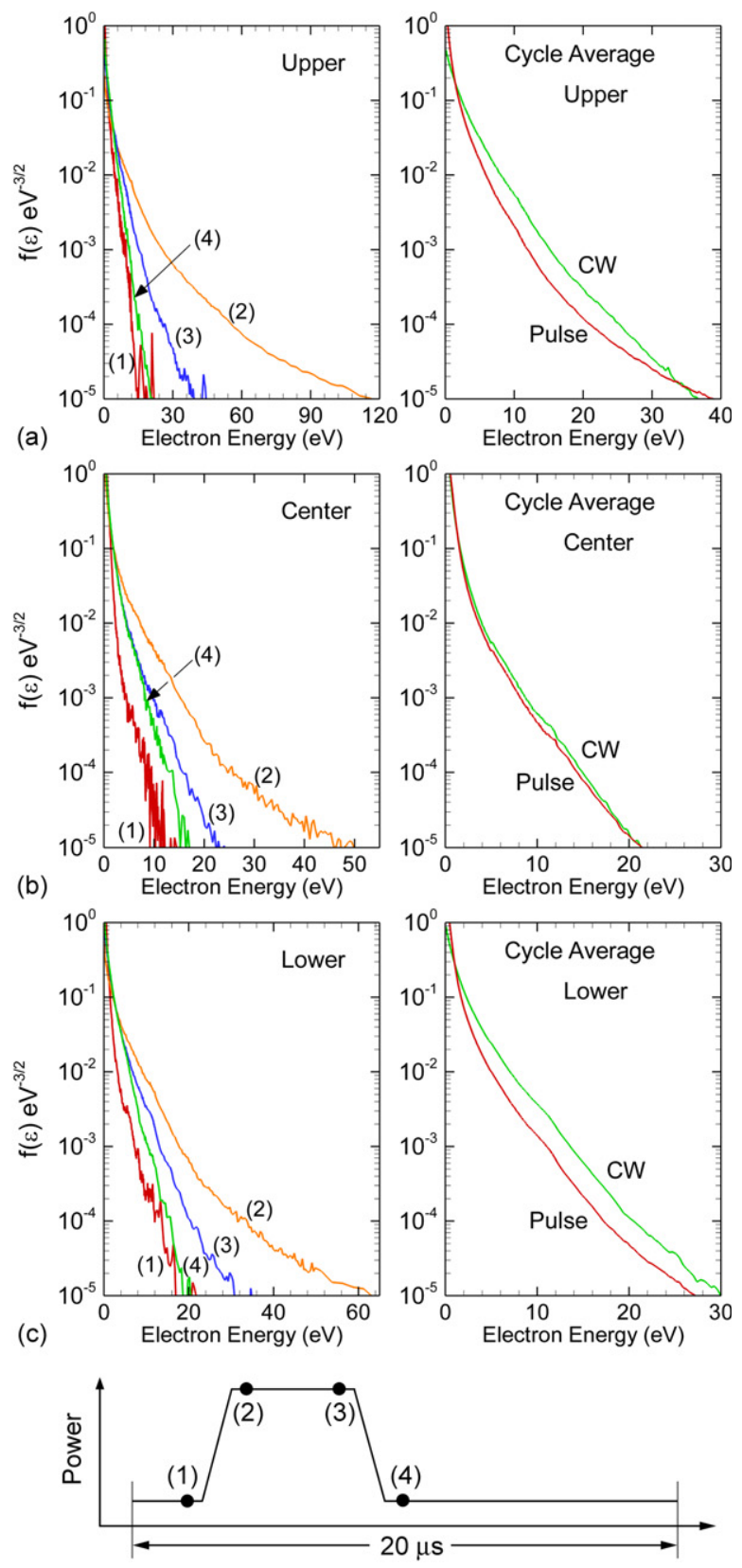

Figure 11. Electron energy distribution functions in $\mathrm{Ar} / \mathrm{CF}_{4} / \mathrm{O}_{2}=75 / 20 / 5$ for the base case conditions (40 mTorr, $500 \mathrm{~W}$ at $10 \mathrm{MHz} \mathrm{CW}$ and $500 \mathrm{~W}$ at $40 \mathrm{MHz}$ in pulse mode- $50 \mathrm{kHz} \mathrm{PRF}$ with $25 \% \mathrm{DC}$ ) at different times during the pulsed cycle (as indicated in the lower figure). (a) Near the HF sheath, $(b)$ in the bulk plasma and $(c)$ near the LF sheath (locations indicated in figure 1). Comparisons of $f(\varepsilon)$ averaged over the pulse period and with $\mathrm{CW}$ excitation are in the right column.

period due to the resonant cross sections for attachment which increase with increasing $E / N$ (at low $E / N$ ).

The source and loss rate coefficients at different heights in the reactor are shown in figure 13. The electron source rate coefficient is dependent on the tail of $f(\varepsilon)$ and so is most sensitive to local sources of electron heating. The ionization rate coefficient is largest near the $\mathrm{HF}$ electrode due to the higher efficiency of stochastic heating, next highest near the LF electrode and lowest in the bulk plasma. The absolute
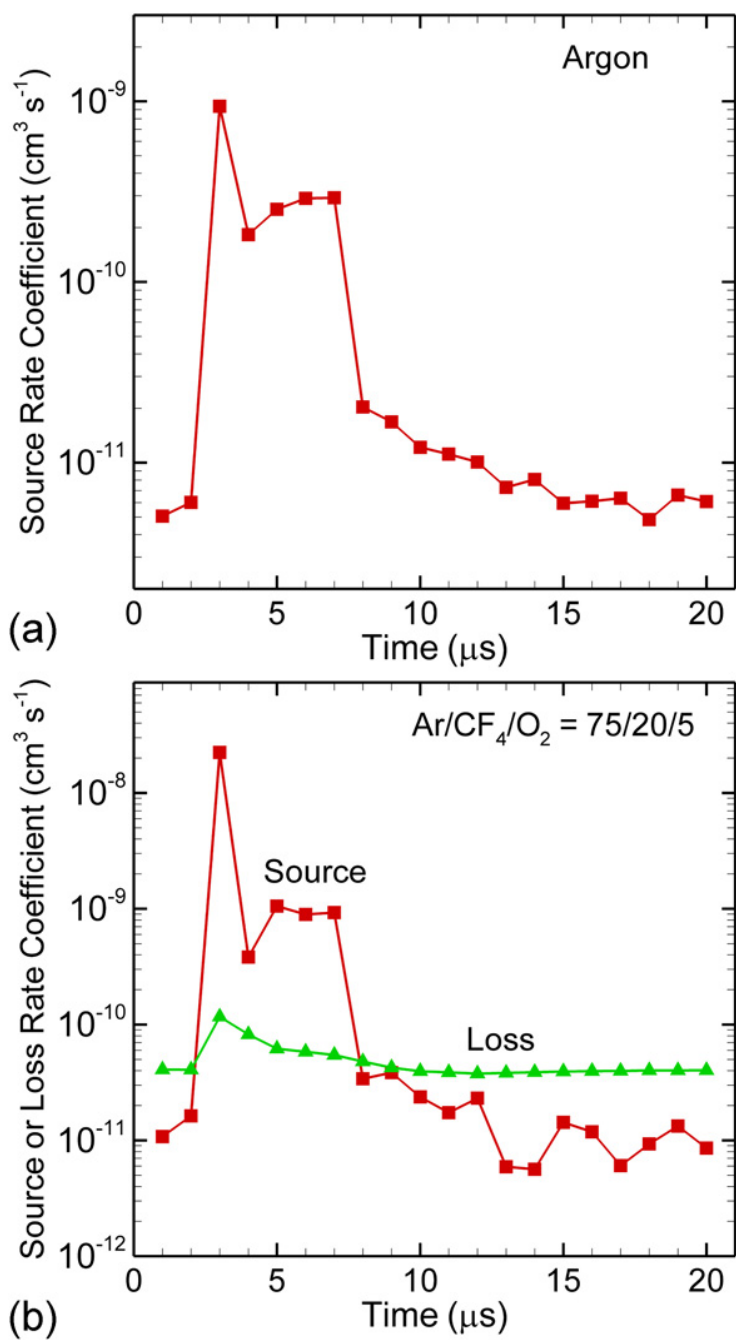

Figure 12. Mole fraction weighted rate coefficients for electron-impact ionization sources and electron-impact loss reactions during the pulsed cycle for the base case conditions. (a) $\mathrm{Ar}$ and (b) $\mathrm{Ar} / \mathrm{CF}_{4} / \mathrm{O}_{2}=75 / 20 / 5$. In Ar, the electron loss rate coefficient is negligible, whereas in $\mathrm{Ar} / \mathrm{CF}_{4} / \mathrm{O}_{2}$ the loss rate coefficient is finite through the pulsed cycle.

value of the source rate coefficients is larger in $\mathrm{Ar} / \mathrm{CF}_{4} / \mathrm{O}_{2}$ than in $\mathrm{Ar}$ in order to compensate for the volumetric electron losses.

There are two electron heating mechanisms-stochastic heating due to the oscillating sheath boundary and Ohmic heating due to the resistivity of the plasma in the bulk. The relative overshoot of the ionization rate coefficient at the beginning of the pulse-on period is largest in the center of the plasma. This is a consequence of long-mean-free path transport of electrons which were accelerated by stochastic heating in the sheaths but which have ionizing collisions in the middle of the plasma-the local value of $E / N$ is not high enough to support the local increase in ionization by Ohmic heating. The electron loss rate coefficients for $\mathrm{Ar} / \mathrm{CF}_{4} / \mathrm{O}_{2}$ behave similarly to the ionization rates, though over a smaller dynamic range. Due to the resonant cross sections for attachment having non-zero threshold energies, the electron loss rates increase during the power-on period-more so near the HF and LF sheaths. 

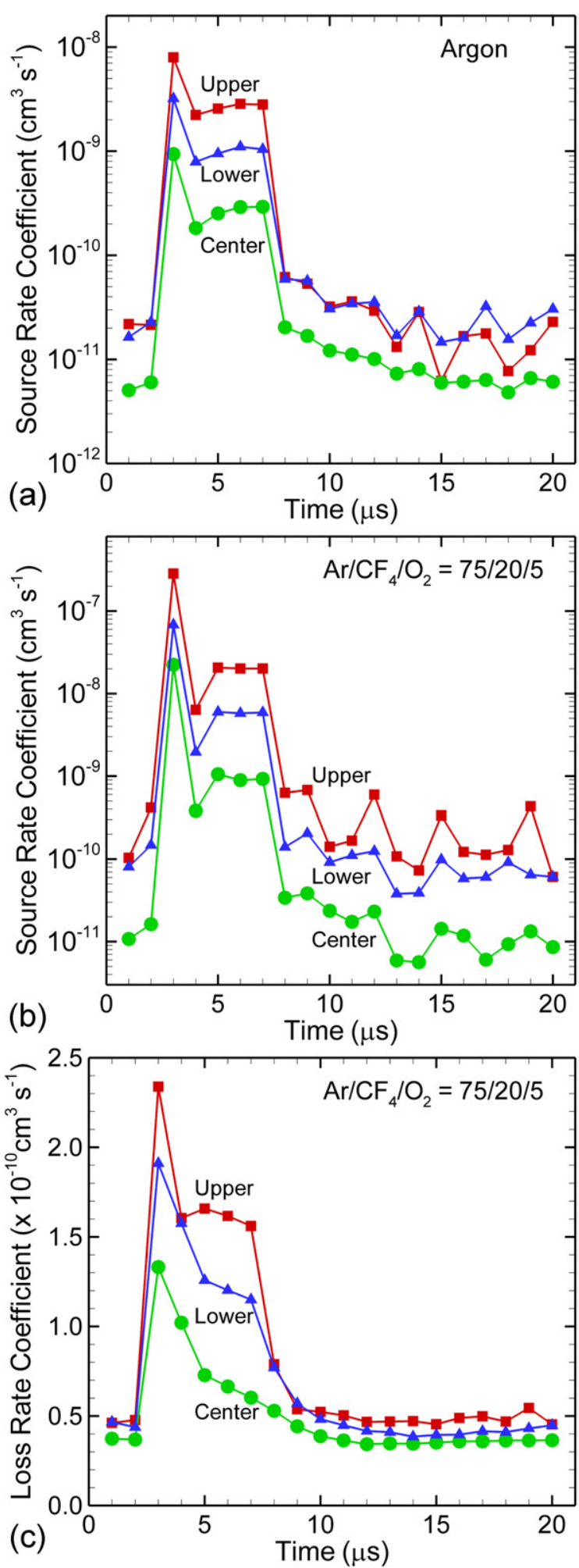

Figure 13. Mole fraction weighted rate coefficients for the electron-impact ionization sources and electron-impact loss reactions during the pulsed cycle at different heights in the reactor for the base case conditions. (a) Ar ionization, sources, $(b)$ $\mathrm{Ar} / \mathrm{CF}_{4} / \mathrm{O}_{2}$ ionization sources and $(c) \mathrm{Ar} / \mathrm{CF}_{4} / \mathrm{O}_{2}$ losses. The loss rate coefficients respond to the overshoot in $E / N$ due to the resonant cross sections for attachment. The locations for heights are shown in figure 1 .

\section{Pulse repetition rate and DC}

The $f(\varepsilon)$ at the beginning of the power-on stage near the HF sheath for different PRFs of 50 and $250 \mathrm{kHz}$, and for $\mathrm{CW}$ excitation sustained in argon and $\mathrm{Ar} / \mathrm{CF}_{4} / \mathrm{O}_{2}$ are shown in figure 14. In both argon and $\mathrm{Ar} / \mathrm{CF}_{4} / \mathrm{O}_{2}$ mixtures, the tail of $f(\varepsilon)$ reaches higher energies with lower PRF. With the lower PRF and longer interpulse period, electron losses are larger and so the conductivity of the plasma is lower at the time the pulse power is applied. This affects collisional heating by there being a larger $E / N$ in the bulk plasma and affects stochastic heating by increasing the sheath width and hence the sheath speed. $f(\varepsilon)$ in $\mathrm{Ar} / \mathrm{CF}_{4} / \mathrm{O}_{2}$ are more distinctly biMaxwellian compared with Ar due to the generation of highenergy electrons during the enhanced overshoot in $E / N$ and the more rapid rate of collisional energy loss (and attachment) in the molecular gas mixture at energies $<12 \mathrm{eV}$ (inelastic threshold for ground state Ar). As the tail is enhanced, the low-energy portion of $f(\varepsilon)$ is more depleted with smaller PRF.

The overshoot of the average electron source rate coefficient at the beginning of the power-on stage is observed in both argon and $\mathrm{Ar} / \mathrm{CF}_{4} / \mathrm{O}_{2}$. However, the relative amount of overshoot decreases with increasing PRF, approaching CW, as shown in figure 15. For a given DC, with increasing PRF, there is less electron loss during the pulse-off period and so a higher conductivity at the start of the next power-on period. The higher conductivity results in a lower $E / N$.

The same logic produces a dependence of $f(\varepsilon)$ on DC. The $f(\varepsilon)$ at the leading edge of the pulse power near the HF sheath for DCs of 25\%,50\% and CW (PRF $=50 \mathrm{kHz}$ ) for argon and $\mathrm{Ar} / \mathrm{CF}_{4} / \mathrm{O}_{2}$ are shown in figure 16. Corresponding ionization coefficients are in figure 17. With decreasing DC and longer interpulse period, there is greater loss of electrons and so smaller conductivity at the start of the power-on period. As a result, the overshoot in $E / N$ is greater and so the tail of $f(\varepsilon)$ extends to higher energy. For a PRF of $50 \mathrm{kHz}$ in argon, the overshoot effect already diminishes with a $50 \% \mathrm{DC}$, whereas, for $\mathrm{Ar} / \mathrm{CF}_{4} / \mathrm{O}_{2}$, the overshoot effect starts to diminish with a DC of $90 \%$, since the electron density is still small compared with argon discharge. The low-energy portion of $f(\varepsilon)$ is enhanced with increasing DC, as shown in the insets of figure 16 , as the tail of $f(\varepsilon)$ decreases. The ionization source rate coefficients reflect two trends with decreasing DC-increasing overshoot in $E / N$ and the increase in power during the power-on portion of the cycle to keep the cycle-averaged HF power constant at $500 \mathrm{~W}$. Note that the ionization rate coefficient increases during the power-on period with argon, but is constant or slightly decreasing with $\mathrm{Ar} / \mathrm{CF}_{4} / \mathrm{O}_{2}$. This is due in part to the accumulation of Ar metastable states during the poweron pulse that provides more efficient ionization by multistep processes, a process that is of less importance in $\mathrm{Ar} / \mathrm{CF}_{4} / \mathrm{O}_{2}$.

\section{Concluding remarks}

The properties of $f(\varepsilon)$ in pulse powered DF-CCP sources sustained in $\mathrm{Ar}$ and $\mathrm{Ar} / \mathrm{CF}_{4} / \mathrm{O}_{2}$ mixtures have been computationally investigated using results from a $2 \mathrm{D}$ plasma hydrodynamic model having an electron Monte Carlo 

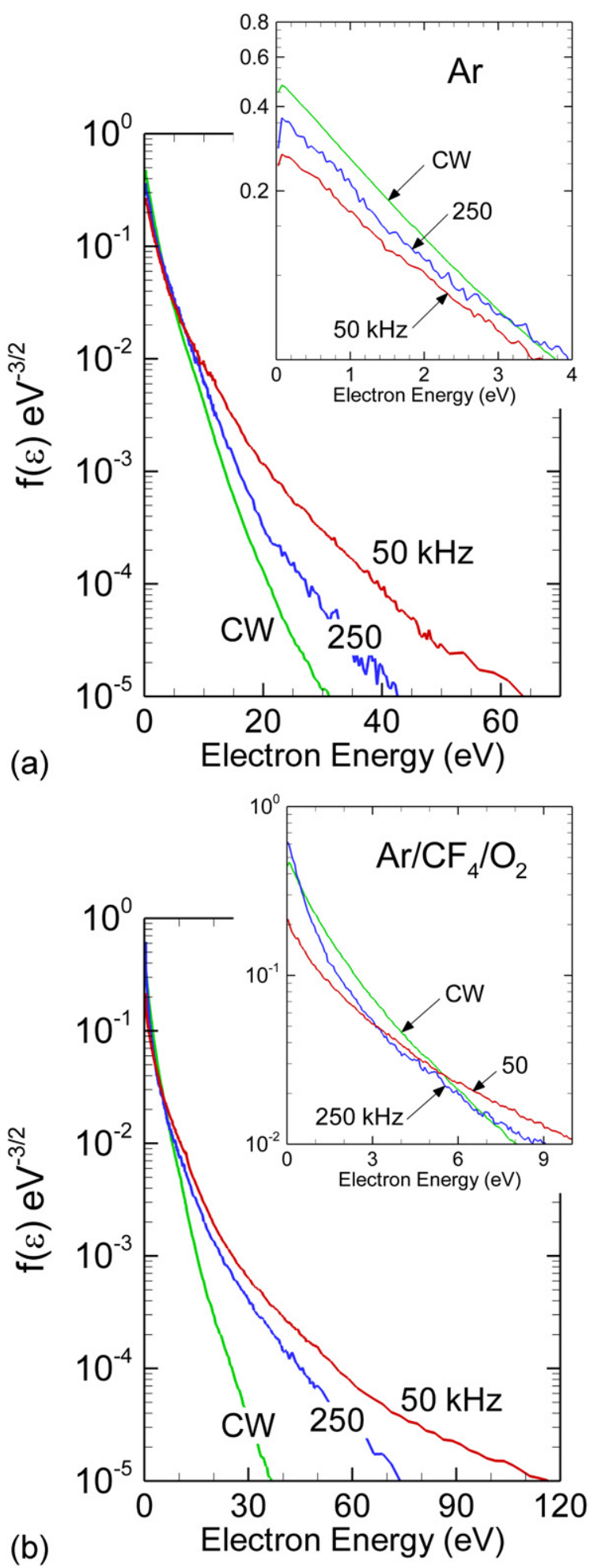

Figure 14. Electron energy distribution functions near the HF sheath for the base case conditions $(40 \mathrm{mTorr}, 500 \mathrm{~W}$ at $10 \mathrm{MHz}$ $\mathrm{CW}, 500 \mathrm{~W}$ at $40 \mathrm{MHz}$ in pulse mode, $25 \% \mathrm{DC}$ ) for different PRFs. (a) Argon and (b) $\mathrm{Ar} / \mathrm{CF}_{4} / \mathrm{O}_{2}$. The insets show enlargements of the low-energy portion of $f(\varepsilon)$.

simulation including electron-electron collisions. The PPA $f(\varepsilon)$ obtained when pulsing the HF power differ from that obtained with $\mathrm{CW}$ excitation and have a shape that arguably would be difficult to replicate under $\mathrm{CW}$ conditions. The

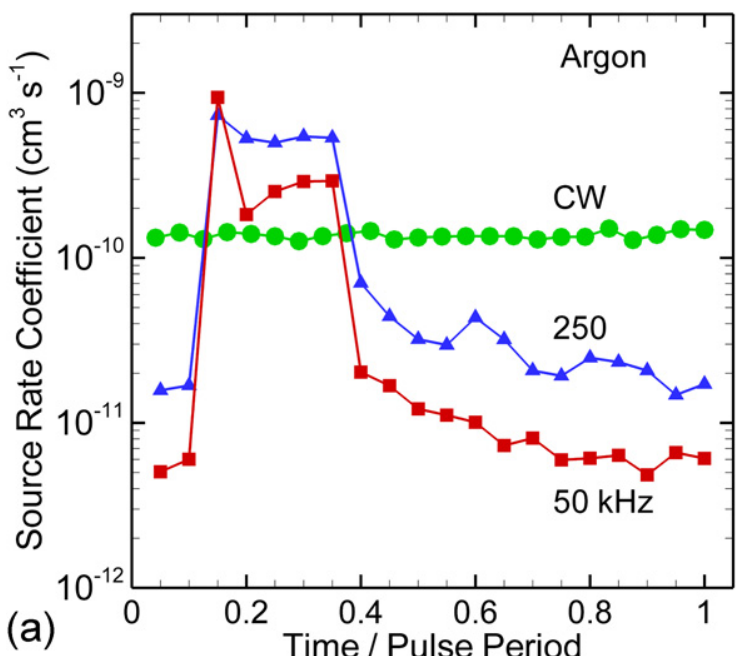

(a)

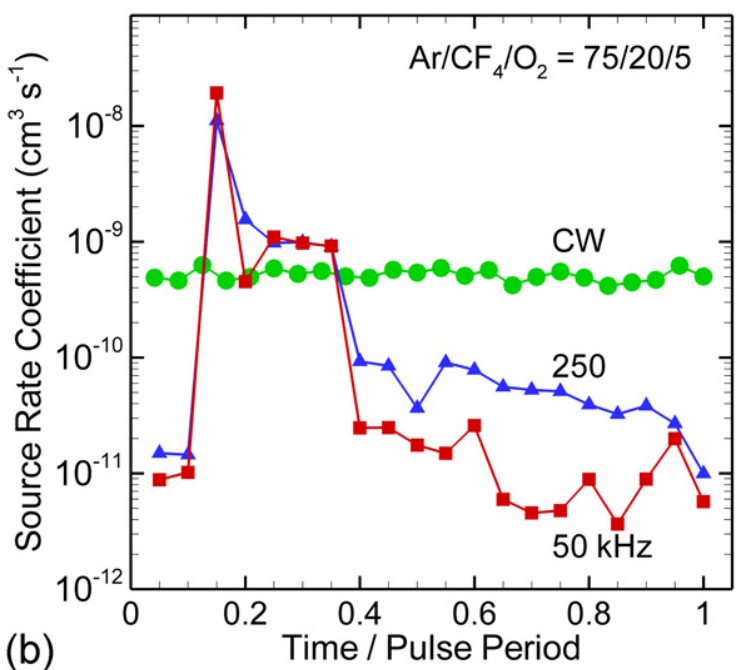

Figure 15. Mole fraction weighted electron-impact ionization rate coefficients in the middle of the gap with different PRFs (40 mTorr, $500 \mathrm{~W}$ at $10 \mathrm{MHz}$ in $\mathrm{CW}$ mode and $500 \mathrm{~W}$ at $40 \mathrm{MHz}$ in pulse mode with $25 \%$ DC). (a) $\mathrm{Ar}$ and (b) $\mathrm{Ar} / \mathrm{CF}_{4} / \mathrm{O}_{2}=75 / 20 / 5$. The relative overshoot of the electron-impact ionization rate coefficient is larger with smaller PRF.

PPA $f(\varepsilon)$ poorly represent the dynamics of $f(\varepsilon)$ during the pulsed period, where the tail of $f(\varepsilon)$ can extend to energies in excess of $100 \mathrm{eV}$ at the leading edge of the pulse-on period. The properties of $f(\varepsilon)$ are differentiated between the HF and LF sheaths, and the bulk plasma. When the power is turned on, the electrons are quickly heated due to the increase in sheath voltage and so sheath width which provides an impulsive acceleration through stochastic heating. The heating is also more prominent at the leading edge of the pulse due to an overshoot of $E / N$ above the quasi-steady state during the pulse-on period. The overshoot is more prominent in $\mathrm{Ar} / \mathrm{CF}_{4} / \mathrm{O}_{2}$ mixtures due to the greater fractional decrease in electron density during the power-off stage. Also, the shorter energy relaxation length in $\mathrm{Ar} / \mathrm{CF}_{4} / \mathrm{O}_{2}$ mixture produces more dynamic changes in the plasma properties near the sheath as the pulse power is toggled on and off. We found that the plasma properties including $f(\varepsilon)$ can be controlled with different PRFs and DCs. Lower PRF and smaller DC produce larger excursions of the tail of $f(\varepsilon)$ and so larger ionization 

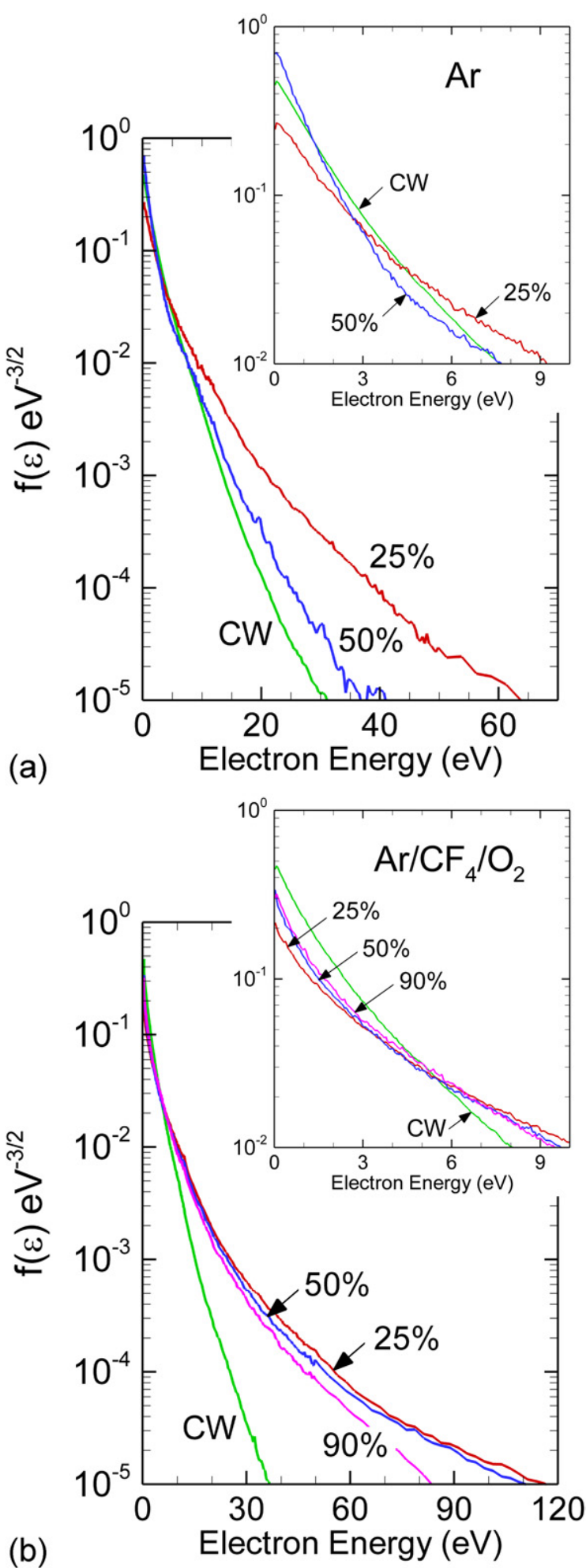

Figure 16. Electron energy distribution functions near the HF sheath for the base case conditions $(40 \mathrm{mTorr}, 500 \mathrm{~W}$ at $10 \mathrm{MHz}$ $\mathrm{CW}, 500 \mathrm{~W}$ at $40 \mathrm{MHz}$ in pulse mode, $50 \mathrm{kHz}$ ) for different DCs. (a) Argon and $(b) \mathrm{Ar} / \mathrm{CF}_{4} / \mathrm{O}_{2}$. The insets show enlargements of the low-energy portion of $f(\varepsilon)$.

sources in both $\mathrm{Ar}$ and $\mathrm{Ar} / \mathrm{CF}_{4} / \mathrm{O}_{2}$ mixtures. These results are sensitive to the electron emitting boundary conditions. With lower values of $\gamma$, more ionization must be provided by bulk electron collisions and so the tail of the $f(\varepsilon)$ is raised. This

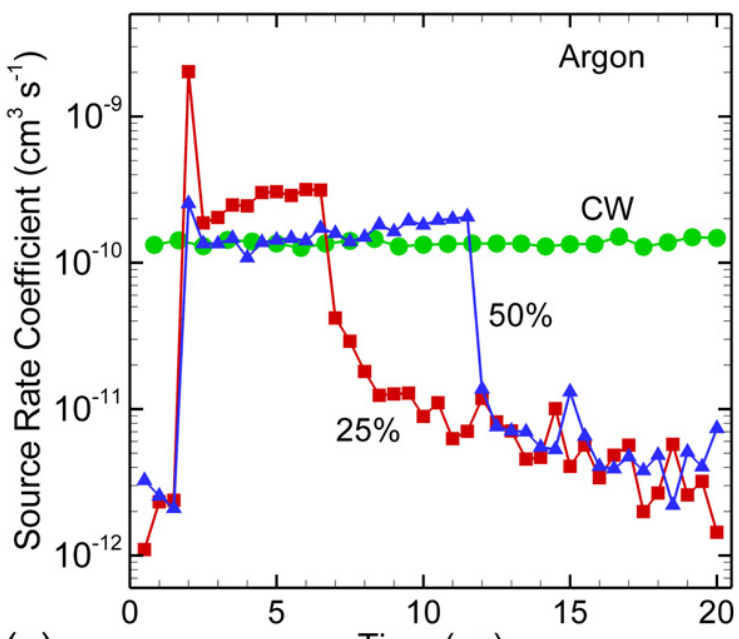

(a) Time $(\mu \mathrm{s})$

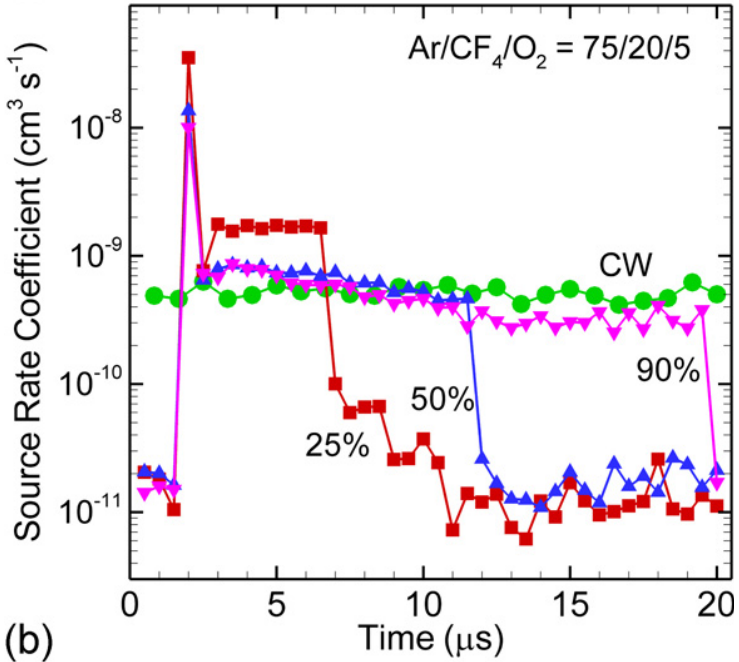

Figure 17. Mole fraction weighted electron-impact ionization rate coefficients in the middle of the gap with different DCs $(40$ mTorr, $500 \mathrm{~W}$ at $10 \mathrm{MHz}$ in $\mathrm{CW}$ mode and $500 \mathrm{~W}$ at $40 \mathrm{MHz}$ in pulse mode, $50 \mathrm{kHz}$ ). (a) Ar and (b) $\mathrm{Ar} / \mathrm{CF}_{4} / \mathrm{O}_{2}=75 / 20 / 5$. The relative overshoot of the electron-impact ionization rate coefficient scales inversely with DC.

gives some opportunity to customize $f(\varepsilon)$ in CCPs by varying the electron emitting boundary condition.

\section{Acknowledgments}

This work was supported by the Department of Energy Office of Fusion Energy Sciences, the Semiconductor Research Corp. and the National Science Foundation.

\section{References}

[1] Sugai H, Ghanashev I, Hosokawa M, Mizuno K, Nakamura K, Toyoda H and Yamauchi K 2001 Electron energy distribution functions and the influence on fluorocarbon plasma chemistry Plasma Sources Sci. Technol. 10378

[2] Godyak V A, Piejak R B and Alexandrovich B M 1992 Measurement of the electron energy distribution in low pressure RF discharges Plasma Sources Sci. Technol. 136 
[3] Blackwell D D and Chen F F 2001 Time-resolved measurements of the electron energy distribution function in a helicon plasma Plasma Sources Sci. Technol. 10226

[4] Godyak V A and Piejak R B 1990 Abnormally low electron energy and heating-mode transition in a low-pressure argon rf discharge at $13.56 \mathrm{MHz}$ Phys. Rev. Lett. 65996

[5] Heason D J and Bradley J W 2001 Modifying the IEDFs at a plasma boundary in a low-pressure RF discharge using electron beam injection Plasma Sources Sci. Technol. 10627

[6] Chung C W, Kim S S and Chang H Y 2004 Experimental measurement of the electron energy distribution function in the radio frequency electron cyclotron resonance inductive discharge Phys. Rev. E 69016406

[7] Hong J I, Seo S H, Kim S S, Yoon N S, Chang C S and Chang H Y 1999 Electron temperature control with grid bias in inductively coupled argon plasma Phys. Plasmas 61017

[8] Akashi H, Samukawa S, Takahashi N and Sasaki T 1997 Dependence of frequency and pressure on electron energy distribution functions in low pressure plasma Japan. $J$. Appl. Phys. 36877

[9] Mareska A, Orlov K and Kortshagen U 2002 Experimental study of diffusive cooling of electrons in a pulse inductively coupled plasma Phys. Rev. E 65056405

[10] Hebner G A and Fleddermann C B 1997 Characterization of pulse-modulated inductively coupled plasmas in argon and chlorine J. Appl. Phys. 822814

[11] Banna $S$ et al 2009 Inductively coupled pulsed plasmas in the presence of synchronous pulsed substrate bias for robust, reliable, and fine conductor etching IEEE Trans. Plasma Sci. 371730

[12] Samara V, Bowden M D and Braithwaite N St J 2010 Effect of power modulation on properties of pulsed capacitively coupled radiofrequency discharges J. Phys. D: Appl. Phys. 43124017

[13] Kushner M J 2009 Hybrid modeling of low temperature plasmas for fundamental investigations and equipment design J. Phys. D: Appl. Phys. 42194013

[14] Vasenkov A V and Kushner M J 2002 Electron energy distributions and anomalous skin depth effects in high-plasma-density inductively coupled discharges Phys. Rev. E 66066411

[15] Lin S L and Bardsley J N 1977 Monte Carlo simulation of ion motion in drift tubes J. Chem. Phys. 66435
[16] Opal C P, Peterson W K and Beaty E C 1971 Measurements of secondary-electron spectra produced by electron impact ionization of a number of simple gases J. Chem. Phys. 554100

[17] Mitchner M and Kruger C H 1973 Partially Ionized Gases (New York: Wiley) p 265

[18] Vahedi V, Lieberman M A, DiPeso G, Rognlien T D and Hewett D 1995 Analytic model of power deposition in inductively coupled plasma sources $J$. Appl. Phys. 781446

[19] Bird G A 1994 Molecular Gas Dynamics and the Direct Simulation of Gas Flows (New York: Oxford University Press)

[20] Vasenkov A V, Li X, Oehrlein G S and Kushner M J 2004 Properties of $\mathrm{C}_{4} \mathrm{~F}_{8}$ inductively coupled plasmas: II. Plasma chemistry and reaction mechanism for modeling of $\mathrm{Ar} / \mathrm{c}-\mathrm{C}_{4} \mathrm{~F}_{8} / \mathrm{O}_{2}$ discharges J. Vac. Sci. Technol. A 22511

[21] Kawamura E, Lieberman M A and Lichtenberg A J 2006 Stochastic heating in single and dual frequency capacitive discharges Phys. Plasmas 13053506

[22] Agarwal A, Stout P J, Banna S, Rauf S and Collins K 2011 Recouping etch rates in pulsed inductively coupled plasmas J. Vac. Sci. Technol. A 29011017

[23] Bonham R A 1994 Electron impact cross section data for carbon tetrafluoride Japan. J. Appl. Phys. 334157

[24] Phelps A V 1985 Tabulations of collision cross sections and calculated transport and reaction coefficients for electron collisions with $\mathrm{O}_{2}$ JILA Information Center Report no 28, September

[25] Commisso R J, Fernsler R F, Scherrer V E and Vitkovitsky I M 1982 Electron-beam controlled discharges IEEE Trans. Plasma Sci. PS-10 241

[26] Boness M J W and Center R E 1977 High-pressure pulsed electrical CO laser J. Appl. Phys. $\mathbf{4 8} 2705$

[27] Douglas-Hamilton D H, Feinberg R M and Lowder R S 1975 Experimental and theoretical electron-beam-sustained $\mathrm{CO}_{2}$ laser output at $\sim 200$ and $\sim 300 \mathrm{~K} \mathrm{~J}$. Appl. Phys. 463566

[28] Haas F A, Goodyear A and Braithwaite N St J 1998 Tailoring of electron energy distributions in low temperature plasmas Plasma Sources Sci. Technol. 7471

[29] Ramamurthi B and Economou D I 2002 Two-dimensional pulsed-plasma simulation of a chlorine discharge $J$. Vac. Sci. Technol. A 20467 